Research Paper

\title{
TBLIX and Flot2 form a positive feedback loop to promote metastasis in nasopharyngeal carcinoma
}

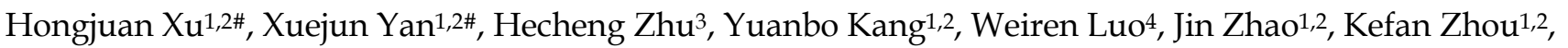
Xiwu Liu'1,2, Li Ye1,2, Quanwei Zhou ${ }^{5}$, Shasha Li ${ }^{1,2}$, Ming Zhao ${ }^{3}$, Lei Wang1,2, Bin Zhu1,2, Weidong Liu ${ }^{1,2}$, Jianxiong Li ${ }^{\circledR}$, Xingjun Jiang ${ }^{\circledR}$, Caiping Ren ${ }^{1,2}$

1. Cancer Research Institute, Department of Neurosurgery, National Clinical Research Center for Geriatric Disorders, NHC Key Laboratory of Biological Nanotechnology, Xiangya Hospital, Central South University, Changsha, Hunan 410008, China.

2. NHC Key Laboratory of Carcinogenesis and the Key Laboratory of Carcinogenesis and Cancer Invasion of the Chinese Ministry of Education, School of Basic Medical Science, Central South University, Changsha, Hunan 410008, China.

3. Changsha Kexin Cancer Hospital, Changsha, Hunan 410008, China.

4. Cancer Research Institute, Shenzhen Third People's Hospital, the Second Affiliated Hospital of Southern University of Science and Technology, Shenzhen, Guangdong 518112, China.

5. Department of Neurosurgery, National Clinical Research Center for Geriatric Disorders, Xiangya Hospital, Central South University, Changsha, Hunan 410008, China.

6. Department of Radiotherapy, Chinese PLA General Hospital, Beijing 100089, China.

\#Equal contributions

$\triangle$ Corresponding author: Caiping Ren: rencaiping@csu.edu.cn, Cancer Research Institute, Department of Neurosurgery, National Clinical Research Center for Geriatric Disorders, NHC Key Laboratory of Biological Nanotechnology, Xiangya Hospital, Central South University, Changsha, Hunan 410008, China. Xingjun Jiang: jiangxj@csu.edu.cn, Department of Neurosurgery, National Clinical Research Center for Geriatric Disorders, Xiangya Hospital, Central South University, Changsha, Hunan 410008, China. Jianxiong Li: 301301ljx@sina.com, Department of Radiotherapy, Chinese PLA General Hospital, Beijing 100089, China.

(c) The author(s). This is an open access article distributed under the terms of the Creative Commons Attribution License (https://creativecommons.org/licenses/by/4.0/). See http://ivyspring.com/terms for full terms and conditions.

Received: 2021.10.15; Accepted: 2021.12.24; Published: 2022.01.01

\begin{abstract}
Metastasis is the main cause of death in patients with nasopharyngeal carcinoma (NPC). The molecular mechanisms underlying the metastasis of NPC remain to be elucidated. TBLIX has been shown abnormally expressed in diverse cancers. However, the role and mechanism of TBLIX in NPC remain unknown. Here, we showed TBLIX expression was significantly higher in metastatic NPC tissues compared to non-metastatic tissues and significantly correlated with TNM stage and metastasis of NPC patients. In addition, NPC patients with high TBLIX expression had a poor prognosis. TBLIX interacted with TCF4 to trans-activate Flot2 expression. TBL1X promoted NPC cell migration and invasion in vitro and in vivo through Flot2. Moreover, Flot2 increased the expression of TBL1X by upregulating c-myc, which was identified to be a positively regulatory transcription factor of TBLIX. TBLIX could restore the functional changes of NPC cells resulting from Flot2 alteration. TBLIX and Flot2 were positively correlated in NPC. Patients with high expression of both TBLIX and Flot2 possessed poorer overall survival (OS) and disease-free survival (DFS) compared to patients with high expression of any single one of the two proteins. Our findings demonstrate that TBL1X and Flot2 positively regulate each other to promote NPC metastasis, which provides novel potential molecular targets for NPC treatment.
\end{abstract}

Key words: Nasopharyngeal carcinoma (NPC); metastasis; prognosis; Transducin $\beta$-like protein 1 X (TBL1X); Flotillin-2 (Flot2)

\section{Introduction}

Nasopharyngeal carcinoma (NPC) is a common head and neck cancer originating from the nasopharyngeal epithelium with about 133,354 new cases and 80,008 related death cases in 2020[1]. Compared with other cancers, NPC shows the uneven geographical distribution, with approximately $70 \%$ of new cases occurring in east and southeast Asia[2].
Because of the concealed anatomical location and lack of specific symptoms during early stages, $70 \%$ of NPC patients are typically diagnosed with locoregionally advanced disease at the initial diagnosis[3]. Metastasis is the primary cause of death in NPC patients[4], thus, it is critical to elucidate the molecular mechanisms of NPC metastasis to identify 
more effective treatment avenues.

TBL1X is encoded by the TBL1X gene located in Xp22.3 genomic region, which contains F-box-like and WD-40-repeat domains, and is identified as a core component of the co-repressor silencing mediator of retinoid and thyroid hormone receptor (SMRT)nuclear receptor co-repressor (N-COR) complex[5, 6]. It has been reported that TBL1X acts as an oncogene to promote the progression of multiple tumors by directly regulating different proteins' expression. For example, TBL1X promotes EMT of breast cancer cells by acting as a cofactor of ZEB1 to regulate the expression of $\mathrm{CDH} 1$, and the high expression of TBL1X contributes to metastasis of breast cancer and is correlated with the poor prognosis of patients with breast cancer[7]. Tegavivint suppresses chemotherapy-resistant and metastatic osteosarcoma by disturbing $\beta$-catenin-TBL1 binding[8], and SUMOylation of TBL1/TBLR1 promotes the migration and invasion of bladder cancer cells[9]. In pancreatic ductal adenocarcinoma, TBL1X acts as a malignancy checkpoint [10]. Nevertheless, the role of TBL1X in NPC metastasis remains unclear.

Flotillin-2 (Flot2) is a lipid raft protein and has been shown to regulate the cancer cell cycle, proliferation, migration, and invasion, which are key processes in cancer development[11, 12]. Upregulation of Flot2 occurs in glioma[13, 14], breast cancer[15], NPC[16], melanoma[17], hepatocellular carcinoma[18], lung cancer[19], and oral squamous cell carcinoma[20]. In the previous study, we used suppressive subtractive hybridization (SSH) to identify that Flot2 is a differentially expressed gene between 5-8F (highly tumorigenic and metastatic) and 6-10B (tumorigenic and non-metastatic) NPC cells, with markedly higher expression in 5-8F cells. Moreover, we found that Flot2 promotes NPC development by activating the NF-kB and PI3K/ Akt3 signaling pathways, and its up-regulated expression induces abnormal cancer cell cycle, apoptosis, migration, and invasion characteristics[21]. However, further studies are needed to elucidate the mechanisms underlying the functions and regulation of Flot2 in NPC.

In this study, we demonstrated the pro-metastatic effects of TBL1X and Flot2 in NPC both in vitro and in vivo and identified the mutual regulation relationship between TBL1X and Flot2. High expression of TBL1X and Flot2 was correlated with reduced survival of NPC patients. Our results suggest that the TBL1X-Flot2 feedback axis contributes to NPC progression and might be a potential molecular target for NPC treatment.

\section{Materials and Methods}

\section{Data acquisition and processing}

To detect the mRNA level of TBL1X in 33 types of cancers, we downloaded the mRNA sequencing data using UCSC Xena (https://xena.ucsc.edu/), containing 33 tumors and 30 types of normal tissues. The expression of TBL1X was evaluated in 30 normal tissues and 33 tumors. Data analysis was conducted using R software (Version 4.0.3) and using "ggpubr" to draw pictures. The expression array data (GSE53819) containing 18 NPC tissues and 18 control tissues, GSE12452 containing 31 NPC tissues and 10 control tissues, and GSE64634 containing 12 NPC tissues and 4 control tissues were downloaded from the Gene Expression Omnibus Database (GEO) (https://www.ncbi.nlm.nih.gov/geo/) for subsequent analysis. After gene reannotation and batch normalization, a new GEO batch containing the three GEO datasets (61 NPC samples, 32 control tissues) was formed and analyzed together.

\section{Tissue specimens}

Firstly, the fresh clinical samples of NPC $(n=40)$ were collected from Xiangya Hospital, Central South University. All patients wrote the informed consent. To further investigate the expression of TBL1X and Flot2 in NPC, tissue microarrays of NPC $(n=126)$ were obtained from Shanghai Super Biotek (Shanghai, China) with permission of the local Institutional Review Board.

\section{Immunohistochemistry (IHC)}

The paraffin-embedded NPC tissues were sectioned into $5 \mu \mathrm{m}$-thick slides. The slides were deparaffinized, performed for antigen retrieval, and blocked with a proper blocking solution. The slides were then incubated with the primary antibody against TBL1X (1:50, \#sc137006, Santacruz) or Flot2 (1:50, \#28208-1-AP, Proteintech). After that, the slides were incubated with relative secondary antibodies and stained with a DAB staining kit (\#ZLI9017, ZSGB BIO, Beijing, China).

\section{Cell lines and cell culture}

Human NPC cell lines (5-8F, 6-10B, c666-1, HK-1, SUNE1, HONE1) were cultured in RPMI 1640 medium supplemented with $10 \%$ fetal bovine serum (FBS) and antibiotics $(100 \mathrm{U} / \mathrm{ml}$ penicillin and $0.1 \mathrm{mg} / \mathrm{ml}$ streptomycin). Normal nasopharyngeal epithelial cells NP69 were cultured in KSFM medium, and normal nasopharyngeal epithelial cells NP460 were cultured in KSFM and Cascade Biologics EpiLife medium (50\%:50\%). All cell lines were incubated at $37^{\circ} \mathrm{C}$ with $5 \% \mathrm{CO}_{2}$ in a humidified atmosphere. 


\section{Gene silencing and overexpression}

Endogenous Flot2 and TBL1X were silenced by small hairpin RNA constructs (pLV-shFlot2, pLV-shTBL1X), and the target sequences are as follows: Flot2: TGTTGTGGTTCCGACTATAAA (1\#), GGTTGTGCAGCGCAAGAAA (2\#); TBL1X: ATGA TCTTCAGGCTCACAATA (1\#), CTTCGACAAGTG CGTCCATAT (2\#); TCF4: CACGAAATCTTCGGA GGACAA (1\#), CAACGGGACAGACAG TATAAT (2\#). pLV-oeFlot2 and pLV-oeTBL1X lentiviral expression plasmids were constructed. pLV-control, pLV-shFlot2, pLV-shTBL1X, pLV-oeTBL1X, and pLV-oeFlot2 plasmids were transfected into 293T cells with psPAX2 and pMD2G plasmids by using Lipofectamine 2000 (Invitrogen). Then, the supernatant of the medium was collected and filtered through a $0.45 \mu \mathrm{m}$ filter. Subsequently, we used the supernatant to infect the cancer cells with polybrene. After $48 \mathrm{~h}$, the positive and stable cell lines were obtained by screening with $2 \mu \mathrm{g} / \mathrm{ml}$ of puromycin.

\section{Western blotting analysis}

Total protein lysates were incubated in RIPA strong lysis buffer with $1 \%$ protease inhibitor cocktail and phosphatase inhibitor for $30 \mathrm{~min}$ on ice, then the supernatant was collected by centrifuging for $30 \mathrm{~min}$ at $4^{\circ} \mathrm{C}, 12,000 \mathrm{rpm}$. The concentration of proteins was measured with the BCA method. $40 \mu \mathrm{g}$ total proteins were separated by using 10\%-12\% sodium dodecyl sulfate-polyacrylamide gel electrophoresis (SDSPAGE). The protein bands were transferred from gel to polyvinylidene difluoride (PVDF) membranes, and the membranes were blocked for $2 \mathrm{~h}$ with $5 \%$ BSA in Tris buffer saline with $0.1 \%$ Tween 20 (TBST) buffer at room temperature. Then, the membranes were incubated with primary antibodies at $4^{\circ} \mathrm{C}$ overnight and washed with TBST buffer (3 times, 6 min each) on the next day. The membranes were subsequently incubated with relevant secondary antibodies for $2 \mathrm{~h}$ at room temperature and visualized with a chemiluminescence reagent (Biosharp). The following antibodies from various vendors were used for studies: anti-TBL1X (1:500, \#13540-1-AP, Proteintech); anti-GAPDH (1:1000, \#SC-47724, Santa Cruz); anti-Flot2 (1:1000, \#3426, Cell Signaling Technology); anti-E-cadherin (1:5000, \#20874-1-AP, Proteintech); anti-c-myc (1:500, \#10828-1-AP, Proteintech); anti-TCF4 (1:1000, \#2569, Cell Signaling Technology); anti-rabbit IgG (A0545) and anti-mouse IgG (A9044) (1:40,000, Sigma).

\section{Quantitative real-time polymerase chain reaction (qRT-PCR)}

Total RNA was extracted from cells with the TRIZOL method. $1 \mu \mathrm{g}$ of RNA were used to synthesize cDNA using RevertAid First Strand cDNA Synthesis Kit (\#K1622, Thermo Scientific). SYBR Green Mix (\#Q111-02, Vazyme) was used for qRT-PCR detection, and GAPDH was used as an internal control. All samples were analyzed in triplicate and the data were calculated according to the $2-\triangle \triangle \mathrm{CT}$ method. The used primers are as follows:

GAPDH forward primer: 5'-CCAGCAAGAGCA CAAGAGGAA-3';

GAPDH reverse primer: 5'-ATGGTACATG ACAAGGTGCGG-3';

TBL1X forward primer: 5'-CAGGGCTCCTTA TGGTGACT-3';

TBL1X reverse primer: 5'-CATATCAGATGCC TCGCAGA-3';

Flot2 forward primer: 5'-GGCTTGTGAGCAG TTTCTGG-3';

Flot2 reverse primer: 5'-TCGAAGGCTCGCTT AGAGTC-3'.

\section{Transwell matrigel invasion assay}

24-well transwell plates $(8-\mu \mathrm{m}$ pore size, Corning) were used for cell invasion assay. 20,000 NPC cells suspended in serum-free medium were plated in the upper chambers coated with matrigel (BD Biosciences), and $0.6 \mathrm{ml}$ of RPMI 1640 medium with $10 \%$ FBS was added to the lower chamber. After incubation for a suitable amount of time, the invaded cells were fixed in $4 \%$ paraformaldehyde, stained by crystal violet, and counted under a microscope.

\section{Wound-healing migration}

For wound-healing migration assays, the cell monolayers were mechanically disrupted using a sterile $200 \mu \mathrm{l}$ pipette tip to generate a linear wound. The average area between the two margins of the wound was measured using Image J Software at suitable time points.

\section{Chromatin immunoprecipitation (ChIP) assay}

ChIP assays were conducted using the ChIP Kit (Abcam 500) according to the manufacturer's protocol. We used the following antibodies to immunoprecipitate the chromatin: anti-TBL1X (\#SC137006, Santacruz); anti-TCF4 (\#2569, Cell Signaling Technology); anti-c-myc (\#10828-1-AP, Proteintech). The precipitated DNA fragments were used to detect the protein-DNA binding results by qPCR as mentioned above and agarose gel electrophoresis (150V, 45min). The relative ChIP primers are as follows:

Flot2 forward primer: 5'-GGGCACCAAGAGG GGGTGCCCAGAC-3';

Flot2 reverse primer: 5'-ATCGGGTTCAGCAT CCTCCCCAGGC-3';

TBL1X forward primer 1: 5'-GTTGCAAGCAG 
ATTCTTG-3';

TBL1X reverse primer 1: 5'-CAAGGAGGTAGT GTATTCTGAGA-3';

TBL1X forward primer 2: 5'-AGGAGAAT CAATCAACCACAATG-3';

TBL1X reverse primer 2: 5'-CAAGGAGTTC CAGAGCGAGAG-3'.

\section{Co-immunoprecipitation (Co-IP) assay}

Total proteins were extracted by NP-40 buffer as mentioned above. The concentration of proteins was measured, and the samples were respectively divided into three parts (Input, IgG, IP). Each part contained about 1-2 mg protein. $20 \mu \mathrm{l}$ protein A/G beads (\#B23201, Bimake, USA) were added to each of the sample parts and the mixture was incubated for $2 \mathrm{~h}$ at $4^{\circ} \mathrm{C}$. Then, IgG or IP antibodies were added to the samples, and the mixture was incubated overnight at $4^{\circ} \mathrm{C}$. The next day, $20 \mu \mathrm{l}$ protein $\mathrm{A} / \mathrm{G}$ beads were added to the samples again and incubated together for $2 \mathrm{~h}$ at $4^{\circ} \mathrm{C}$. Magnetic beads were collected, mixed with NP40 lysis and loading buffer, and denatured at $95^{\circ} \mathrm{C}$ for $5 \mathrm{~min}$. Then the supernatant was collected by centrifugation. At last, protein-protein interaction was detected by western blotting as mentioned above.

\section{Immunofluorescence staining}

Cells were added to the coverslip in 24 well plates. The next day, cells were fixed with $4 \%$ paraformaldehyde for $5 \mathrm{~min}$. 5\% FBS was used to incubate cells for $2 \mathrm{~h}$ at RT. The cells were incubated with primary antibodies overnight at $4^{\circ} \mathrm{C}$, and then incubated with relative FITC or Cy5 labeled secondary antibodies at room temperature for $2 \mathrm{~h}$ in dark. DAPI solution was used to stain the cell nuclear. The staining photos were taken by using Laser Scanning Confocal Microscopy (UltraView, Perkin Elmer, Cambridge, UK).

\section{Dual-luciferase assay}

The TBL1X and Flot2 promoter regions including -2000 bp upstream of the transcription start site or relevant mutant sequences were cloned into the pGL3-basic vector. A dual-luciferase system (Promega, USA) was used to measure firefly and renilla luciferase activities according to the manufacturer's protocol.

\section{Mouse lung metastasis model}

All of the animal experiments were approved by the Animal Care Committee of Central South University following Institutional Animal Care and Use Committee guidelines. To study the effects of Flot2 and TBL1X on NPC metastasis in vivo, the 18-20 g male nude mice (BALB/C) used in this study were purchased from the Animal Research Center of
Central South University. To establish the in vivo lung metastasis model, $200 \mu \mathrm{L}$ blank RPMI 1640 containing $2 \times 10^{6}$ 5-8F control, 5-8F shTBL1X, 5-8FshTBL1X+ oeFlot2, 5-8FshFlot2, or 5-8FshFlot2+oeTBL1X cells were respectively injected into the mice by the tail vein injection ( $\mathrm{n}=6$ per group). After about 2 months, the mice were sacrificed and their lung tissues were detached. All of the dissected tissue samples were paraffin-embedded, sectioned, and stained with HE, and the lung metastases were counted.

\section{Statistical analysis}

All the experimental data were presented as (means $\pm \mathrm{SD}$ ). Analyses were performed by using GraphPad Prism Software 8.0 (San Diego, CA, USA) and SPSS 19.0. Student $t$-test was used for comparing two groups, and One-way ANOVA was applied for comparing multiple groups. Pearson's $X^{2}$ test was used to analyze the relevance of TBL1X and Flot2 expression with clinicopathological characteristics. Spearman correlation coefficient was used to assay the correlation between TBL1X and Flot2. Kaplan-Meier analysis was performed to produce OS and DFS curves, and the log-rank test was used to calculate $P$-values. ${ }^{*} P<0.05,{ }^{* *} P<0.01$ and ${ }^{* * *} P<0.001$ were considered statistically significant.

\section{Results}

\section{TBLIX expression and clinical significance in NPC}

Firstly, we carried out a pan-cancer analysis to investigate the expression of TBL1X in different cancer types and their corresponding normal tissues using TCGA datasets. The results demonstrated that TBL1X expression was significantly different in 13 types of tumors compared to their relative normal tissues, with higher expression of TBL1X in six types of cancers, including HNSC, and lower expression in the other seven types of tumor tissues (Figure 1A). NPC is a common form of HNSC, so we analyzed the expression of TBL1X in NPC microarray by IHC methods. The results showed TBL1X expression was positively correlated with the clinical stages $(P<0.001)$ and metastasis of NPC $(P<0.001)$ (Table 1$)$. Meanwhile, NPC patients with metastasis possessed higher staining intensity than patients with non-metastasis (Figure 1B), and the scores of IHC in the metastatic NPC group were also significantly higher than the non-metastatic group $(P<0.05)$ (Figure 1C). Patients with high TBL1X levels had reduced OS and DFS, compared to those with the low level of TBL1X (Figure 1D-E). Consistent with the IHC results, we detected the mRNA level of TBL1X in fresh NPC samples by qRT-PCR, and the results displayed that the expression of TBL1X was significantly higher in 
metastatic tissues than in non-metastatic tissues from NPC patients $(P=0.0171)$ (Figure $1 F)$. Western blotting showed that TBL1X was upregulated in NPC cell lines
(5-8F, c666-1, 6-10B, HK-1, SUNE1, and HONE1), as compared to normal nasopharyngeal epithelial (NPE) cell lines (NP69 and NP460) (Figure 1G).

A

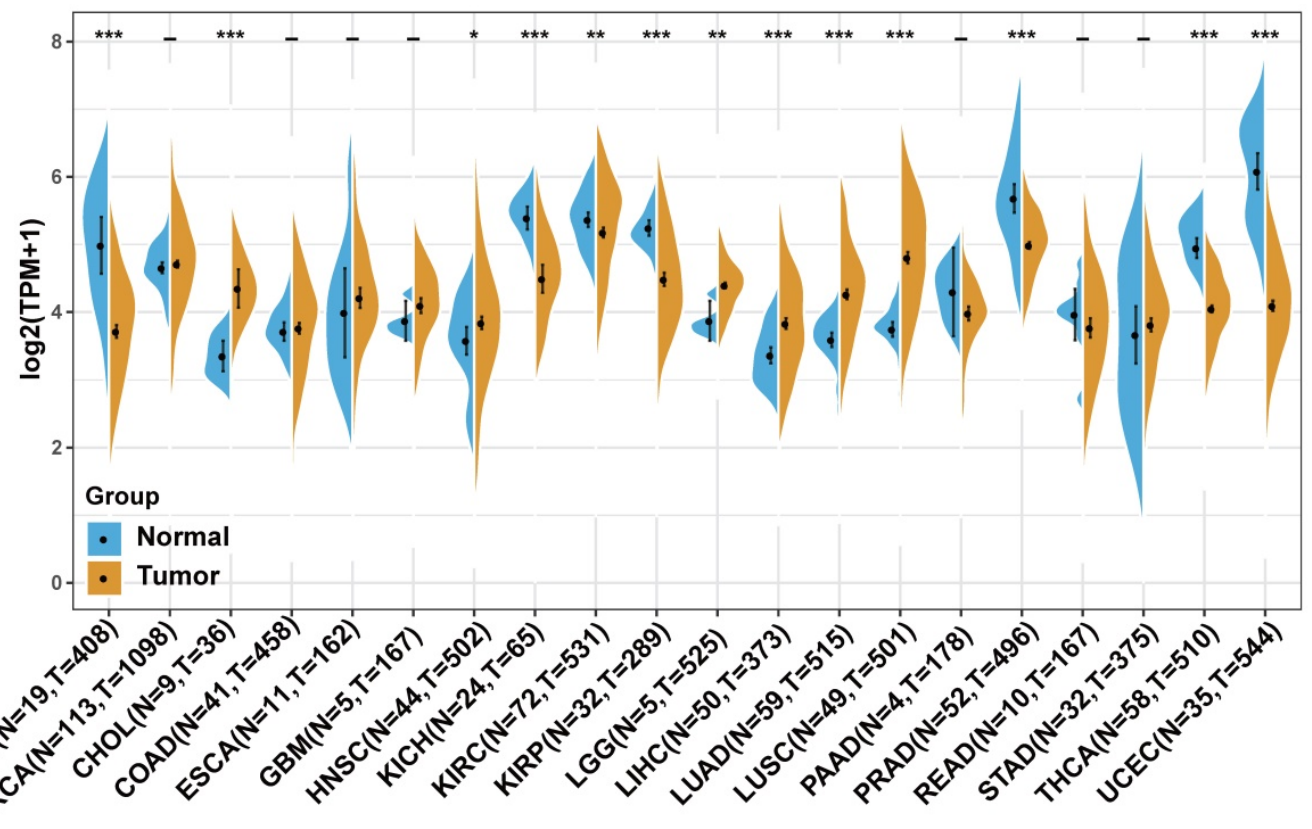

B

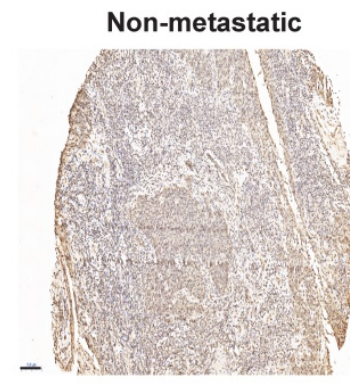

Metastatic

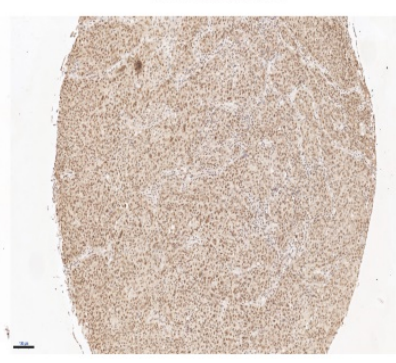

C

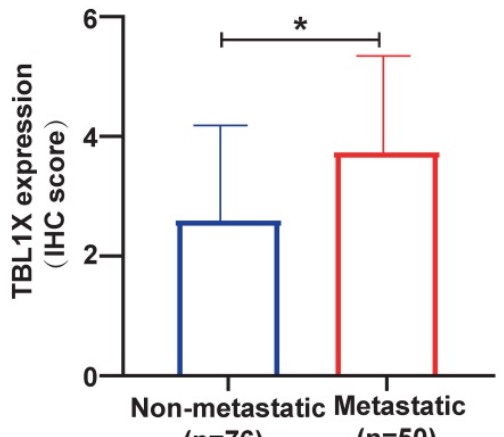

D
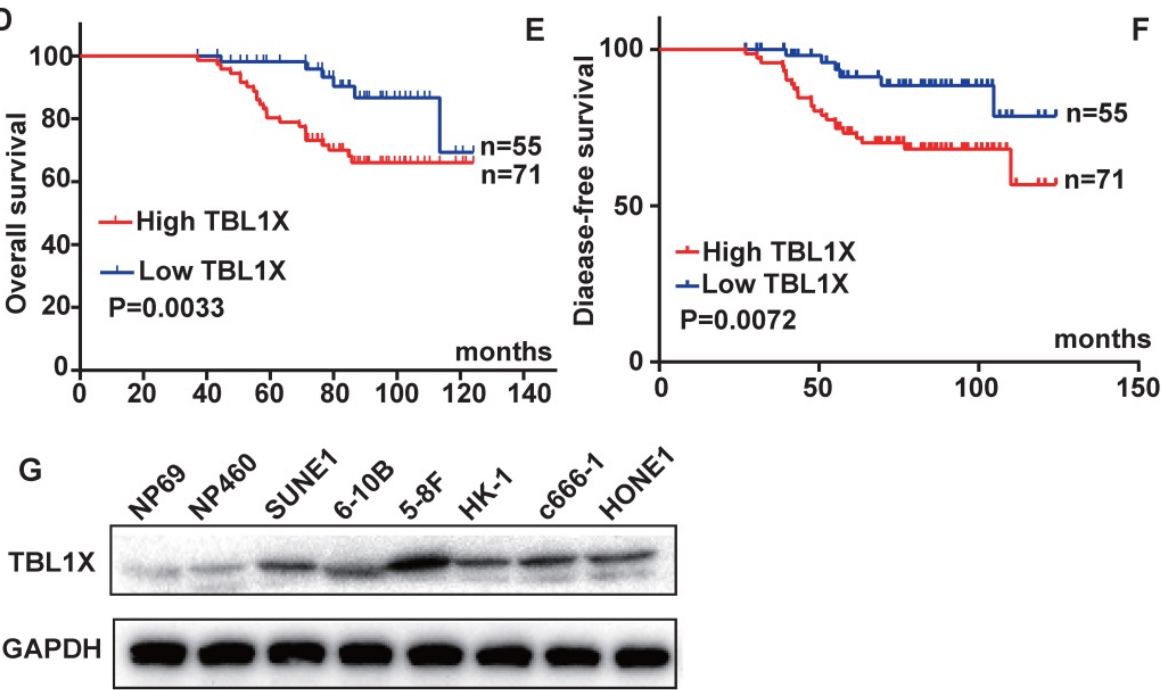

Figure 1. The expression of TBLIX in NPC and its correlation with patient survival. (A) TBLIX expression in different cancers. (B) Representative images of IHC staining of TBLIX in non-metastatic and metastatic NPC tissues (scale bar=100 $\mu \mathrm{m}$ ). (C) The IHC scores of TBLIX expression. (D, E) The OS (D) and DFS (E) curves of NPC patients were associated with TBL1X expression. (F) Analysis of TBLIX mRNA expression in NPC tissues. (G) TBL1X protein expression in NPC cells and normal NPE cells. The results are shown as means \pm SD. $* P<0.05, * * P<0.01, * * * P<0.001$. 
Table 1. Association of TBLIX and Flot2 expression with the clinicopathological characteristics of NPC patients

\begin{tabular}{|c|c|c|c|c|c|c|c|c|c|c|}
\hline \multirow[t]{2}{*}{ Group } & \multirow[t]{2}{*}{$\mathbf{n}$} & \multicolumn{2}{|c|}{ TBL1X expression } & \multirow[t]{2}{*}{$x^{2}$} & \multirow[t]{2}{*}{$P$} & \multirow[t]{2}{*}{$\mathbf{n}$} & \multicolumn{2}{|c|}{ Flot2 expression } & \multirow[t]{2}{*}{$x^{2}$} & \multirow[t]{2}{*}{$P$} \\
\hline & & low(55) & high(71) & & & & $\operatorname{low}(43)$ & high(83) & & \\
\hline \multicolumn{11}{|l|}{ Age } \\
\hline$<52$ & 58 & 23 & 35 & 0.698 & 0.404 & 58 & 21 & 37 & 0.207 & 0.649 \\
\hline$\geq 52$ & 68 & 32 & 36 & & & 68 & 22 & 46 & & \\
\hline \multicolumn{11}{|l|}{ Gender } \\
\hline Male & 81 & 37 & 44 & 0.379 & 0.538 & 81 & 26 & 55 & 0.415 & 0.519 \\
\hline Female & 45 & 18 & 27 & & & 45 & 17 & 28 & & \\
\hline \multicolumn{11}{|c|}{ TNM stage } \\
\hline I+II & 77 & 45 & 32 & 17.61 & $\left.P<0.001^{a}\right)$ & 77 & 32 & 45 & 4.864 & $0.027 a)$ \\
\hline $\mathrm{III}+\mathrm{IV}$ & 49 & 10 & 39 & & & 49 & 11 & 38 & & \\
\hline \multicolumn{11}{|c|}{ Metastasis } \\
\hline Yes & 50 & 9 & 41 & 22.174 & $\left.P<0.001^{a}\right)$ & 50 & 11 & 39 & 5.4223 & $0.02^{a)}$ \\
\hline No & 76 & 46 & 30 & & & 76 & 32 & 44 & & \\
\hline
\end{tabular}

a): The values between groups have a statistically significant difference

\section{TBLIX enhances migration and invasion of NPC cells by regulating the EMT pathway}

To investigate the potential function of TBL1X in NPC cells, we established 5-8FshTBL1X, c6661shTBL1X, 6-10B-TBL1X, and HK-1-TBL1X cell lines. Transwell invasion experiments showed that reduced TBL1X expression suppressed the invasion of $5-8 \mathrm{~F}$ and c666-1 cells using two different siTBL1X sequences (Figure 2A, Supplementary Figure 1A), whereas TBL1X overexpression increased the invasion of 6-10B and HK-1 cells (Figure 2B). Scratch tests revealed that knockdown of TBL1X significantly reduced the migration of 5-8F and c666-1 cells (Figure 2C, Supplementary Figure 1B). Meanwhile, TBL1X overexpression enhanced the migration of $6-10 \mathrm{~B}$ and HK-1 cells (Figure 2D). Consistent with these results, western blotting showed that TBL1X knockdown increased E-cadherin expression and decreased Vimentin expression (Figure 2E, Supplementary Figure 1C). By contrast, TBL1X overexpression resulted in decreased E-cadherin expression and increased Vimentin expression (Figure 2F). These results indicated that TBL1X affects the invasion and migration of NPC cells via altering the EMT pathway of NPC cells.

\section{TBLIX promotes EMT by enhancing Flot2 expression}

To investigate the potential molecular mechanisms underlying the functions of TBL1X in NPC, we classified all tumor samples into high and low expression groups based on the median of TBL1X expression in the TCGA datasets. Firstly, we conducted GSEA to identify pathways that were differentially activated between low and high TBL1X expression groups. Results showed that low TBL1X expression significantly correlated with adhesion molecules (Figure 3A). Meanwhile, high TBL1X expression was positively related to the Wnt signaling pathway (Figure 3B). As a transcription factor, TCF4 is a key component of Wnt signaling. In this study, immunofluorescence staining and Co-IP experiments showed that TBL1X and TCF4 formed protein-protein complexes in NPC cells (Figure 3C-D). Therefore, TBL1X might regulate the expression of downstream genes along with TCF4. We screened out the possible genes that were regulated by TBL1X and TCF4 through CHEV, ENCODE, JASPAR, MotifMap, TRANSFAC websites. Then, we conducted a deeply functional enrichment analysis to explore the potential function of the related genes and found that among the top 20 terms of gene ontology cellular component, the most significant item was adhesion junction. Of interest, Flot2 was located in several GO terms, such as adhesion junction, plasma membrane protein complex, cytoplasmic region, membrane region, membrane raft, and membrane microdomain (Figure 3E). These results suggested that Flot2 might be involved in the TBL1X regulatory network.

To further determine the role of TBL1X in Flot2 expression, we performed qRT-PCR and western blotting tests and the results demonstrated that both mRNA and protein levels of Flot2 were decreased after knockdown of TBL1X or TCF4 using two different siRNA sequences in 5-8F cells (Figure 4A-B, Supplementary Figure 2A-B). Overexpression of TBL1X or TCF4 led to increased Flot2 expression (Figure 4C-D). We then identified a consensus TCF4 binding site in the promoter region of Flot2 using the ALGGEN PROMO and JASPAR online tools (Figure $4 \mathrm{E})$. ChIP assays in $5-8 \mathrm{~F}$ cells were conducted using anti-TBL1X, anti-TCF4 antibodies, and IgG was used as a negative control antibody. The sequence containing the putative TCF4 binding site was amplified. As shown in Figure 4F, the TBL1X/TCF4 complex is directly bound to the Flot 2 promoter region. The Flot2 promoter region fragments, including wild-type or mutated TCF4 binding sites, were inserted into pGL3-basic plasmids to construct gene reporter vectors (Figure 4G). Knockdown of TCF4 or TBL1X genes in 5-8F cells significantly 
decreased luciferase activity in cells transfected with wild-type Flot2 promoter plasmids, but not in cells with mutant Flot2 plasmids (Figure $4 \mathrm{H}$ ). After transfecting TBL1X or TCF4 expressing plasmids, the luciferase activity was significantly increased in 6-10B cells transfected with wild-type Flot2 promoter plasmids, but not in cells transfected with mutant Flot2 plasmids (Figure 4I). These results demonstrated that TBL1X could form a protein-protein complex with TCF4 to increase Flot2 expression at the transcriptional level by binding to the promoter region of the Flot2 gene.
A

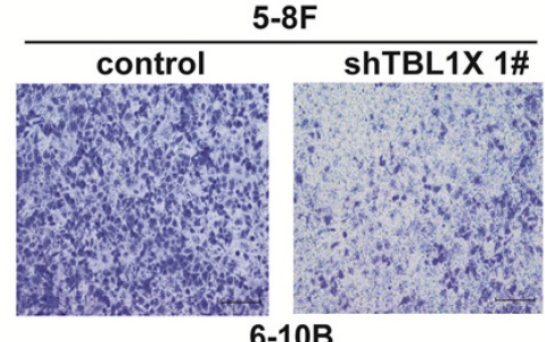

B
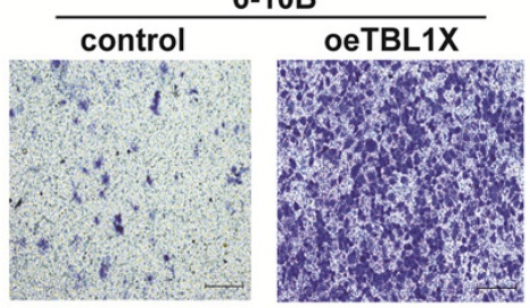

C

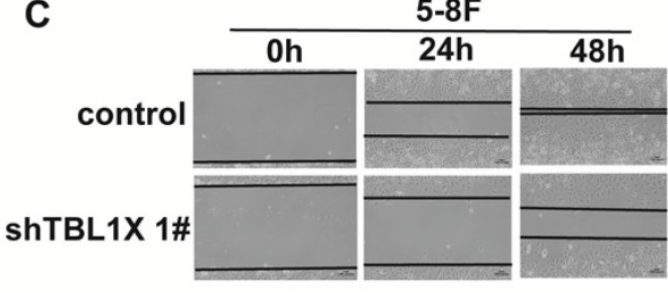

D

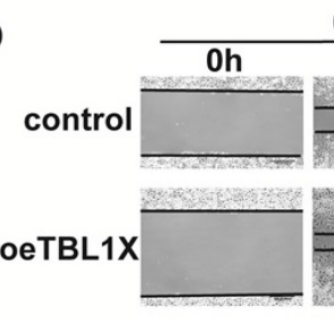

6-10B $24 \mathrm{~h}$

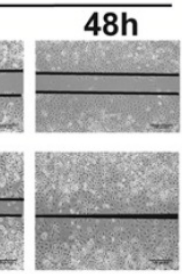

c666-1
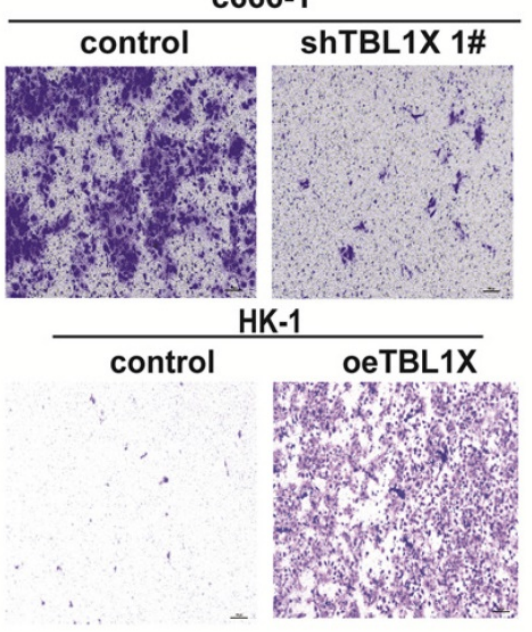

K-1

c666-1

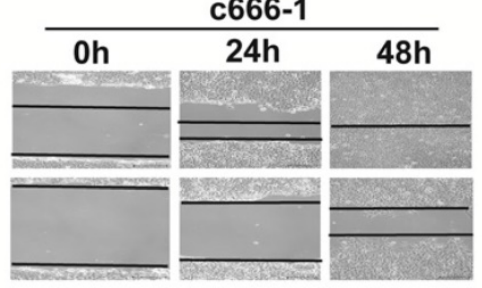

HK-1
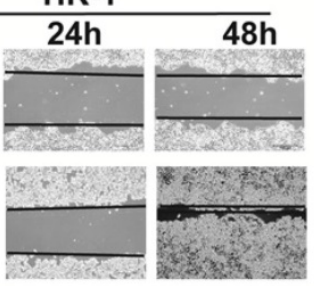
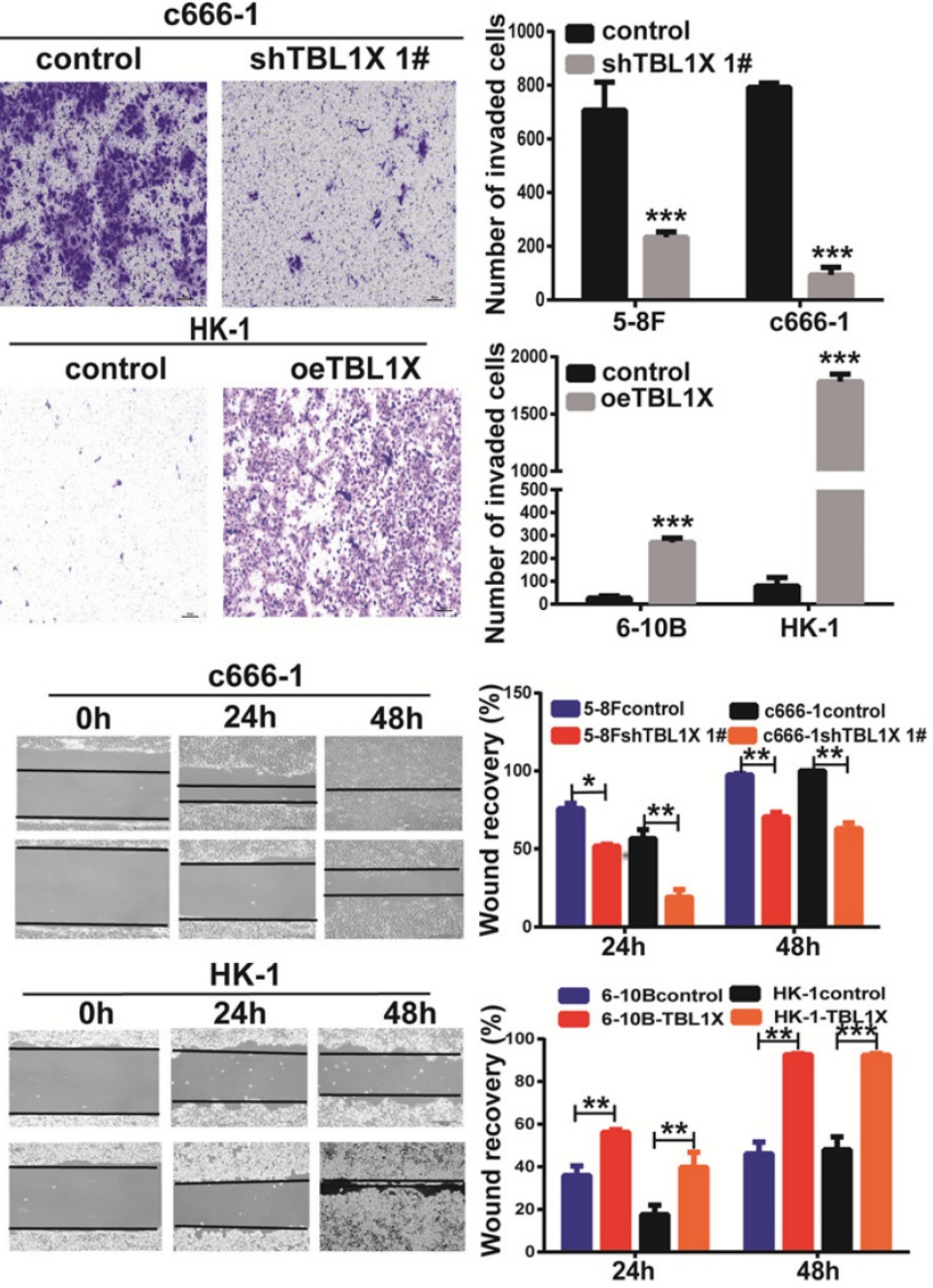

E
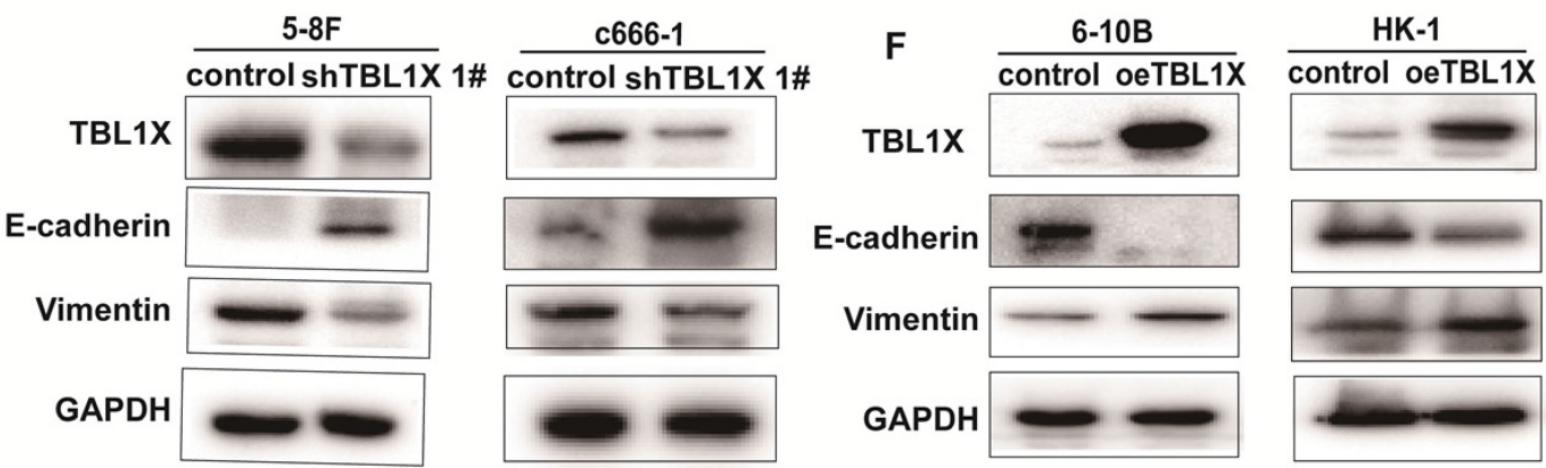

Figure 2. TBLIX enhances NPC cell migration and invasion. (A, B) Transwell invasion assay indicated the invasion abilities after knockdown (A) and overexpression (B) of TBL1X in NPC cells. (C, D) Wound-healing assay indicated the migration abilities after knocking down (C) and overexpression (D) of TBL1X in NPC cells. (E, F) Western blotting results indicated the effects of TBLIX interference $(E)$ and overexpression $(F)$ on EMT markers. The results are shown as means $\pm S D$. $* P<0.05$, $* * P<0.01$, $* * * P<0.001$. 
A

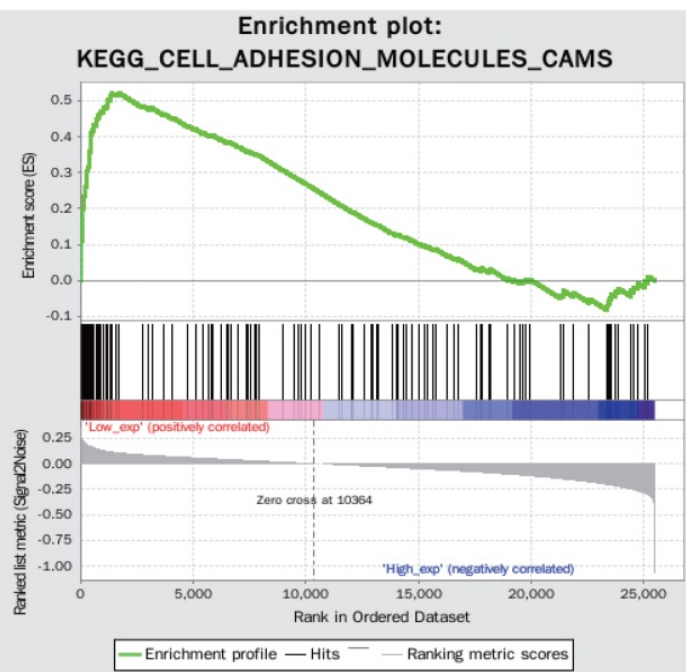

C

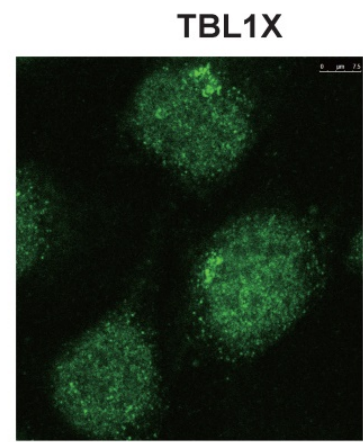

D

\section{Input IgG IP:TBL1X}

IB

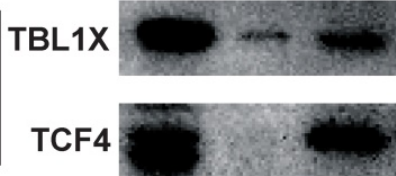

Input IgG IP:TCF4

IB

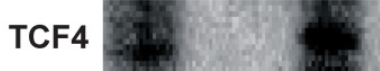

TBL1X

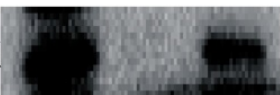

E

\section{B}

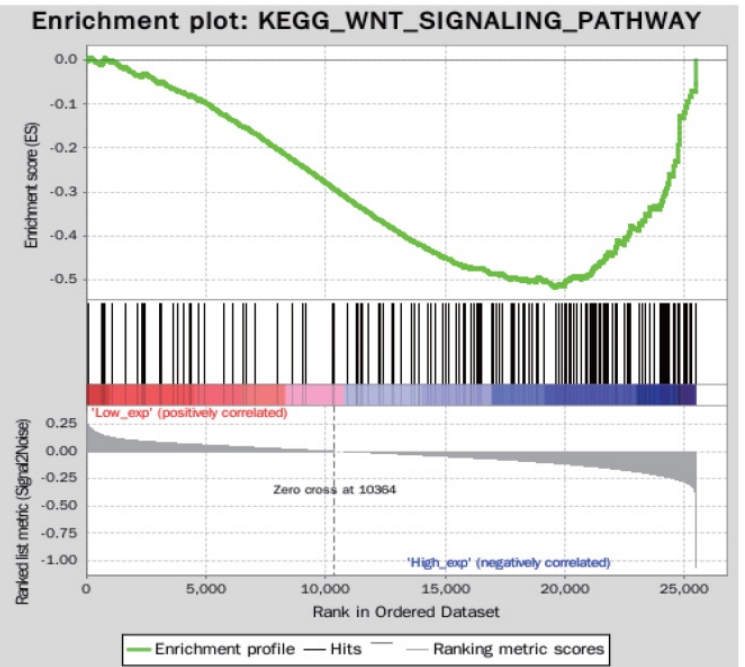

DAPI
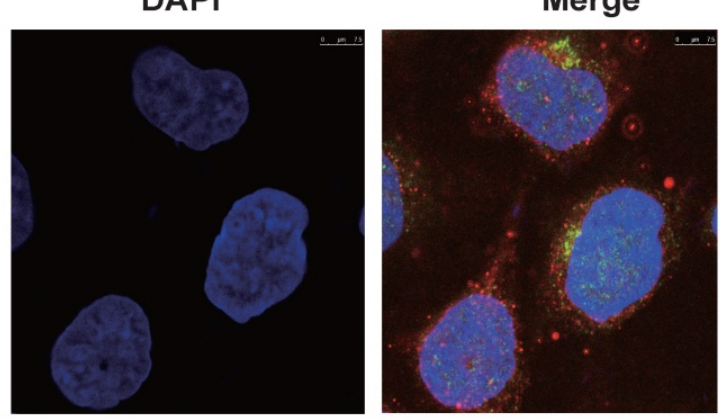

Top20 geneontology_Cellular_Component

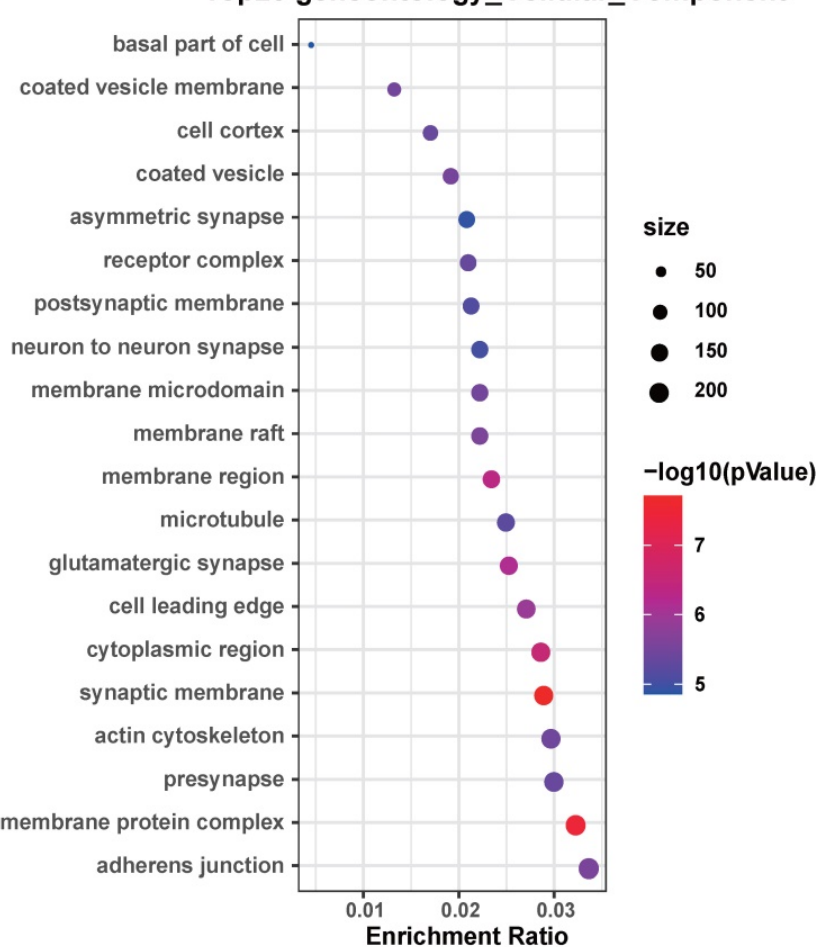

Figure 3. Functional mechanisms of TBLIX in NPC. (A, B) GSEA analysis of TBLIX expression. (C) Immunofluorescence staining showed that TBLIX and TCF4 colocalized in nuclear. (D) Co-IP test demonstrated that TBLIX and TCF4 bound with each other. (E) The top 20 enriched gene ontology cellular component based on KEGG enrichment analysis of TBLIX and TCF4 regulated genes. 
A

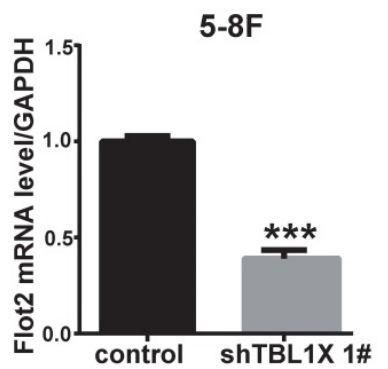

C

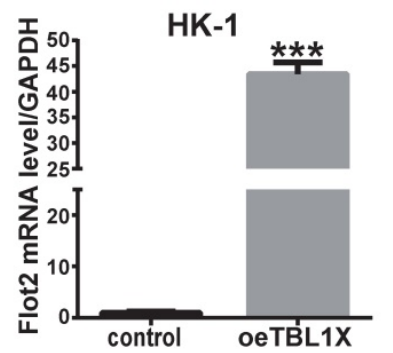

E

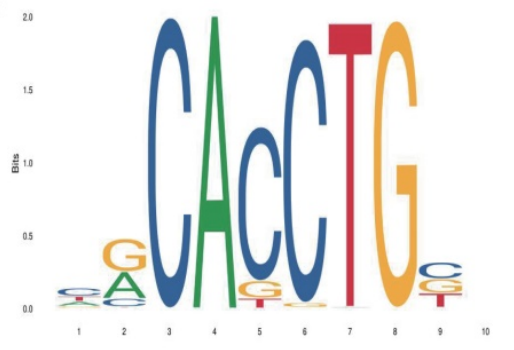

G

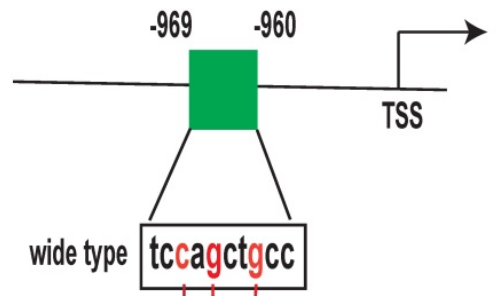

mutant type tcaatctacc

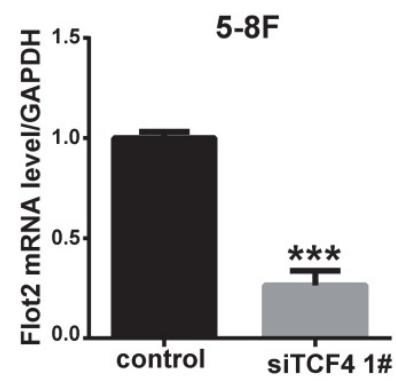

B

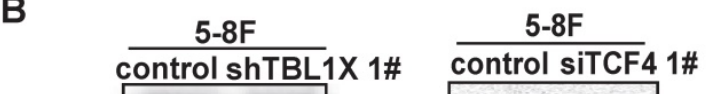

TBL1X

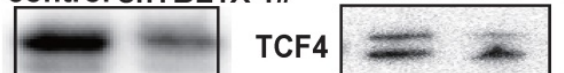

Flot2

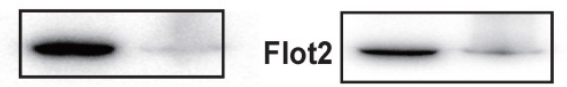

GAPDH

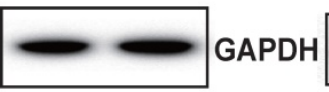

D

HK-1

HK-1

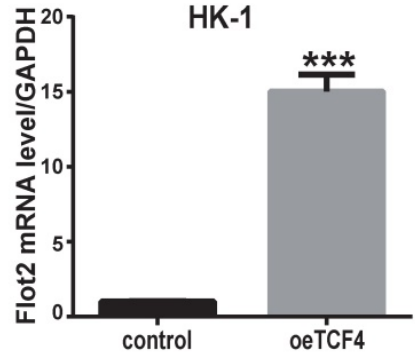

TBL1X

control oeTBL1X controloeTCF4

Flot2

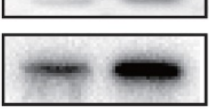

GAPDH

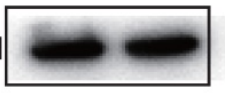

GAPDH

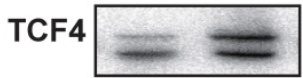

$\mathbf{F}$
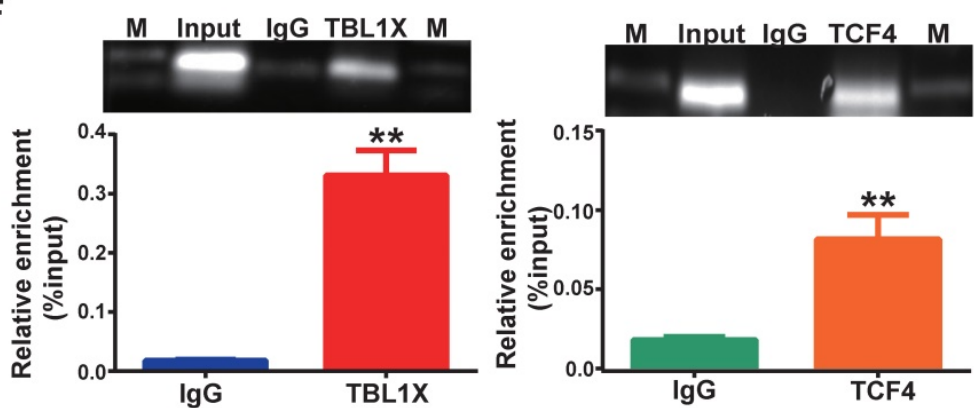

$\mathrm{H}$
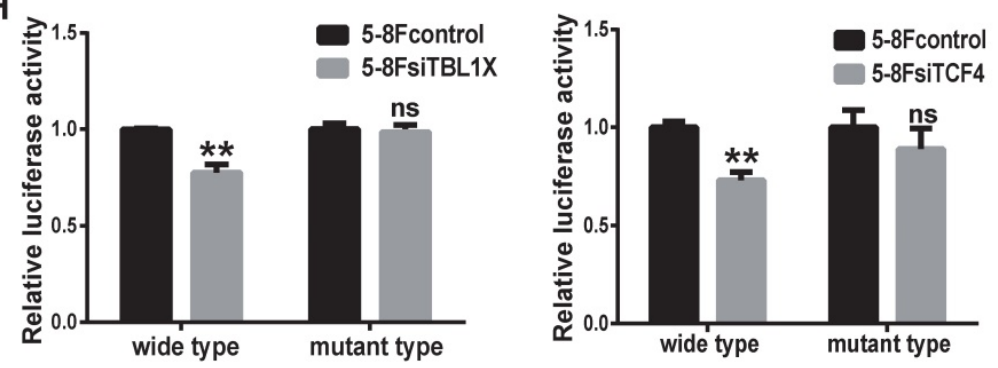

I
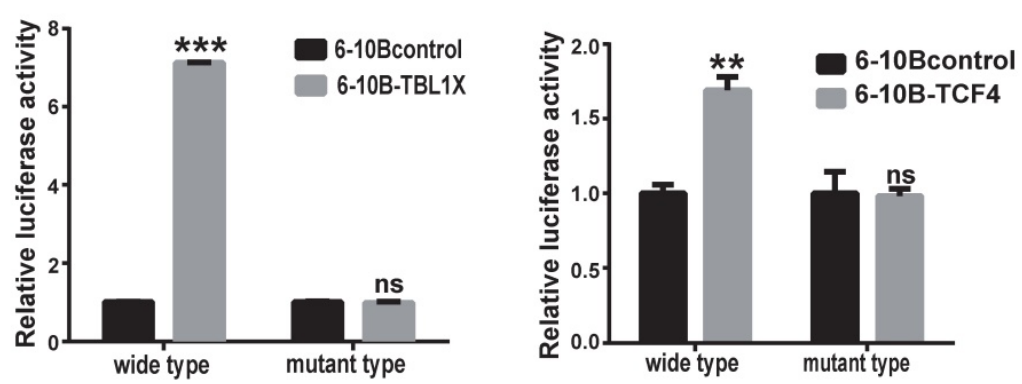

Figure 4. TBL1X regulates Flot2 expression by binding to its promoter with TCF4. (A, B) qRT-PCR (A) and Western blotting (B) showed that knockdown of TBL1X or TCF4 reduced the expression of Flot2. (C, D) qRT-PCR (C) and Western blotting (D) assays showed that overexpression of TBL1X or TCF4 enhanced the expression of Flot2. (E) JASPAR database predicted the binding sites of TCF4 in Flot2 promoter region. (F) ChIP experiments indicated that TBL1X and TCF4 could bind to the promoter region of Flot2. (G) Construction design of dual-luciferase reporter plasmids. (H) Relative luciferase activity in 6-10B cells transfected with pGL3basic-Flot2 plasmids containing wild type or mutant Flot2 promoter and treated with siTBL1X or siTCF4. (I) Relative luciferase activity in 5-8F cells overexpressing TBL1X or TCF4 after transfection of PGL3basic-Flot2 plasmids containing wild type or mutant Flot2 promoter. The results are shown as means \pm SD. $* P<0.05, * * P<0.01, * * * P<0.001$.

From the above experiments, it was shown that Flot2 was regulated by TBL1X. Thus, we analyzed whether Flot2 would reverse the effects of altered TBL1X expression on NPC cell functions. TBL1X 
knockdown cells were transfected with Flot2 expression plasmids. The results showed that Flot2 expression significantly increased the invasion and migration abilities of TBL1X knockdown cells (Figure 5A and 5C). TBL1X-overexpressing cells were transfected with shFlot2 plasmids, which showed that reduced Flot2 levels markedly inhibited TBL1X overexpression-mediated migration and invasion of NPC cells (Figure 5B and 5D). Further, we established a mouse NPC lung metastasis model by injecting 5-8Fcontrol, 5-8FshTBL1X, and 5-8FshTBL1X+oeFlot2 cells by the tail vein injection. The number of pulmonary metastatic nodules was significantly lower in the 5-8FshTBL1X group than in the 5-8Fcontrol group, and this number was markedly higher in the $5-8$ FshTBL1X+oeFlot2 group than in the 5-8F shTBL1X group (Figure 5E-F). The HE staining was consistent with this result (Figure 5G).

\section{Flot2 regulates TBLIX expression through c-myc}

In our previous study, we identified differentially expressed mRNAs in Flot2-depleted NPC cells using a cDNA microarray (GSE67456). The TBL1X gene was one of the downregulated genes in Flot2-knockdown NPC cells. By using qRT-PCR and western blotting methods, we showed that Flot2 knockdown mediated by two different siFlot2 sequences reduced TBL1X expression level, whereas overexpressing Flot2 increased TBL1X level. (Figure 6A-B, Supplementary Figure 3). c-myc is upregulated in NPC[22]. Our results indicated that c-myc expression was reduced after Flot2 knockdown, and Flot2 overexpression increased c-myc protein level (Figure 6B). In 5-8FshFlot2 cells, c-myc overexpression increased TBL1X expression (Figure 6C). c-myc is an important transcription factor. To further investigate whether c-myc directly regulates TBL1X expression, we searched possible binding sites of c-myc in the promoter region of TBL1X gene using the UCSC, PROMO, and JASPAR databases. Two main sites were identified, i.e., site 1 (-885 to -874) and site 2 (-71 to -60 ) (Figure 6D). We then used a ChIP assay to verify their binding to c-myc, and showed that c-myc was more likely to bind to site 1 region than site 2 region (Figure 6E). ChIP results showed that relative TBL1X enrichment was significantly higher in complex precipitated by anti-c-myc than precipitated by IgG (Figure 6F). We then constructed a TBL1X promoter-luciferase reporter plasmid by inserting the $2 \mathrm{~kb}$ of $5^{\prime}$ promoter region of TBL1X into the pGL3-basic plasmid as well as a version with mutated c-myc binding sites (Figure 6G). Luciferase activity was significantly reduced in $5-8 \mathrm{~F}$ cells transfected with wild-type plasmids after c-myc depletion, but not in cells with mutant-type plasmids (Figure 6H). c-myc overexpression increased luciferase activity in 6-10B cells transfected with wild-type plasmids, but not in cells transfected with mutant plasmids (Figure 6I). Taken together, these results demonstrated that Flot2 increased TBL1X expression at the transcriptional level by regulating the expression of c-myc, which is the transcription factor of TBL1X.

Considering TBL1X is regulated by the Flot2-c-myc axis, and c-myc can directly regulate TBL1X expression as a transcription factor, we thus investigated whether TBL1X can rescue NPC cell functions after altering Flot2 levels. 5-8FshFlot2 cells were transfected with TBL1X expression plasmids, the results indicated a significantly higher invasion and migration abilities of 5-8FshFlot2+oeTBL1X cells than those of 5-8FshFlot2 cells (Figure 7A and 7C). Furthermore, 6-10B-Flot2 cells were transfected with shTBL1X plasmids, and reduction of TBL1X expression markedly reduced Flot2 overexpressionmediated migration and invasion abilities of NPC cells (Figure 7B and 7D). Meanwhile, we found that the metastatic lung nodules in nude mice of the shFlot2 group were significantly less than those in controls, which were partly increased in the shFlot2+oeTBL1X group (Figure 7E-F). The HE staining was consistent with this result (Figure 7G). The above results suggested that the TBL1X-Flot2 feedback axis promoted NPC metastasis in vivo.

\section{TBLIX expression is positively correlated with Flot2 expression in NPC}

The above results suggested a TBL1X-Flot2 feedback axis that could influence NPC metastasis. We then investigated the potential prognostic values of the TBL1X-Flot2 feedback axis. Firstly, we examined the correlations of TBL1X and Flot2 in NPC. IHC results showed both high TBL1X and Flot2 levels in NPC tissues (Figure 8A). A Spearman test showed that TBL1X was positively correlated with Flot2 in NPC samples of the GEO batch (61 NPC samples) $(\mathrm{R}=0.3209 ; P=0.0117)$ (Figure 8B). Based on the IHC score and Kaplan-Meier survival analyses, NPC patients with high expression of Flot2 showed reduced OS and DFS (Figure 8 C). Flot2 expression level was significantly correlated with the tumor node metastasis (TNM) and tumor metastasis; no significant correlation between Flot2 level and gender or age was observed (Table 1). More importantly, NPC patients with both high TBL1X and high Flot2 expression showed more significantly reduced OS and DFS compared to patients with the high level of only one of the two proteins (Figure 8D). 

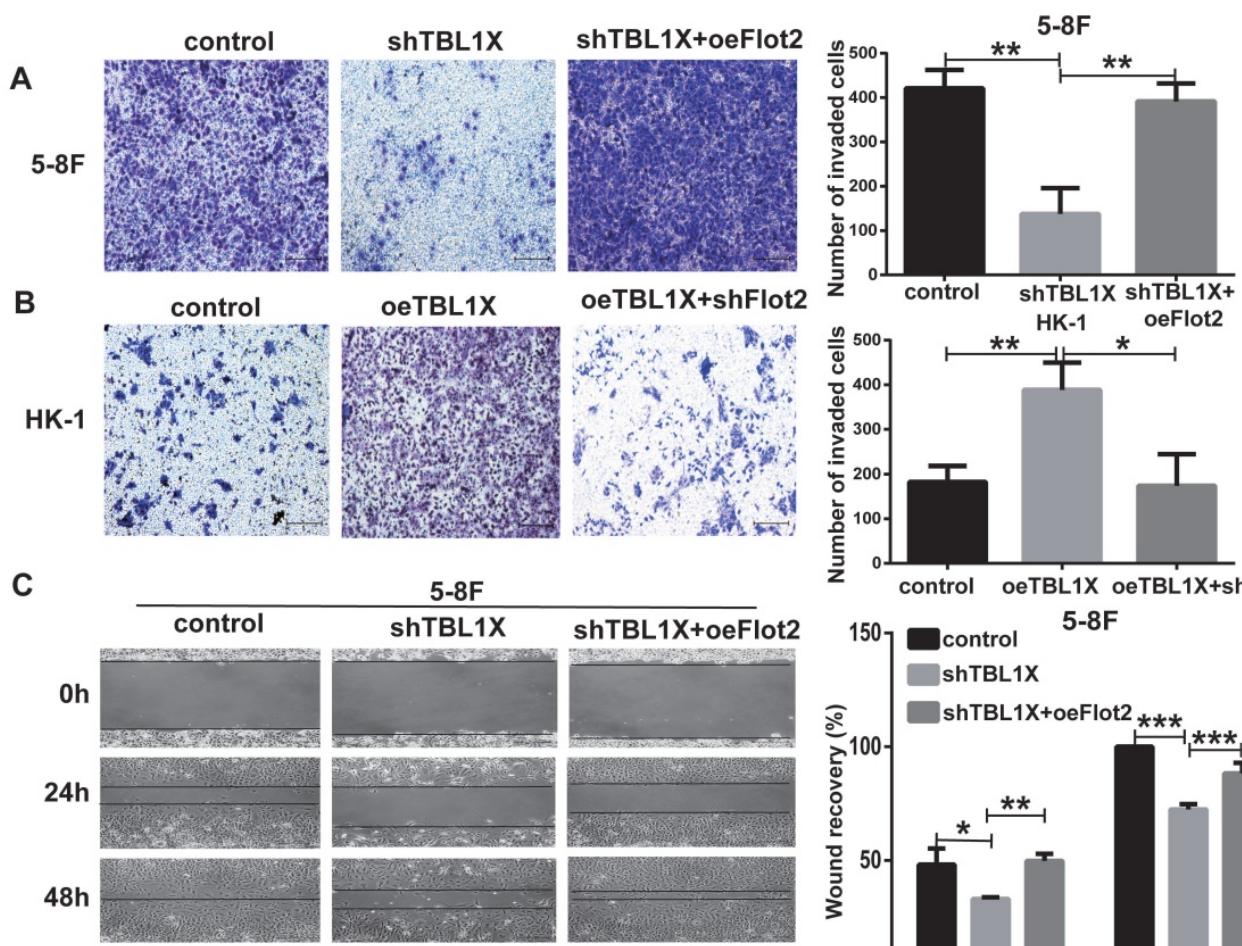

$5-8 \mathrm{~F}$

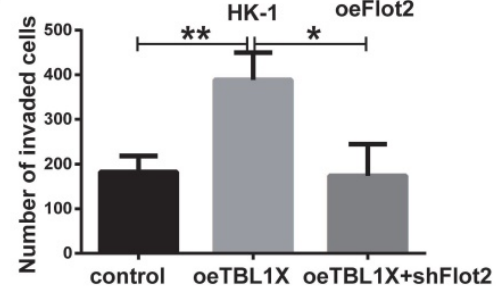

D
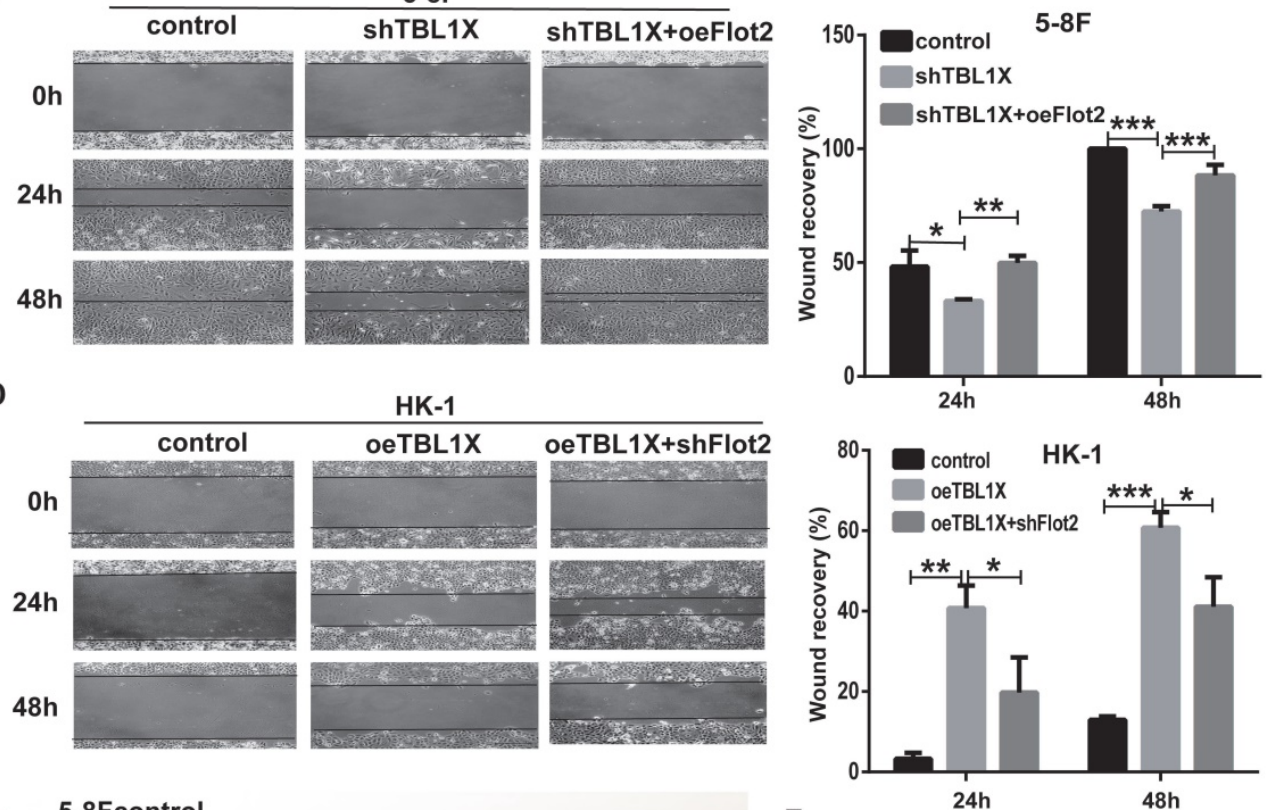

E
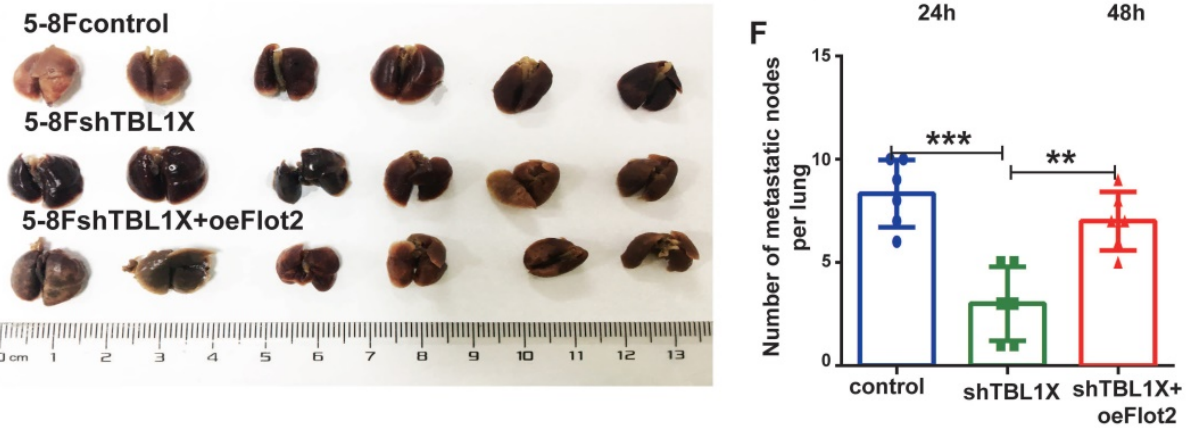

G

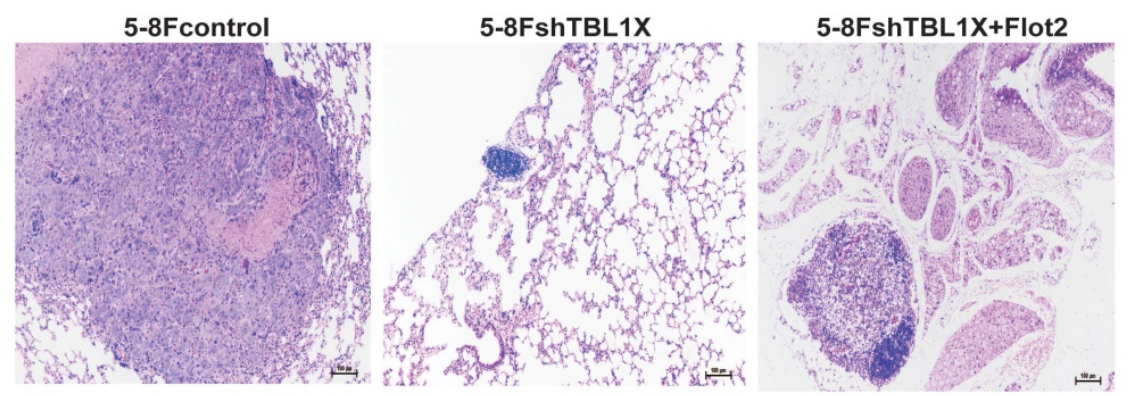

Figure 5. Flot2 rescues the effects of altered TBLIX expression on NPC cells in vitro and in vivo. (A) Matrigel invasion analysis showed that oeFlot2 enhanced the invasion of 5-8FshTBL1X cells. (B) shFlot2 inhibited the invasion of HK-1-TBL1X cells. (C) Wound-healing assay showed that oeflot2 enhanced the migration of 5-8FshTBL1X cells. (D) shFlot2 reduced the migratory ability of HK-1-TBL1X cells. (E) Nude mouse metastasis assay showed the lung metastases via tail vein injection of 5-8Fcontrol, $5-8 F$ shTBLIX or 5-8FshTBL1X+oeflot2 cells $(n=6$, respectively). (F) The number of lung metastases in mice. (G) Representative HE staining images of lung metastases resulting from 5-8Fcontrol, 5-8FshTBLIX and 5-8FshTBLIX+oeFlot2 cell inoculation. The results are shown as means \pm SD. $* P<0.05, * * P<0.01, * * * P<0.001$. 
A
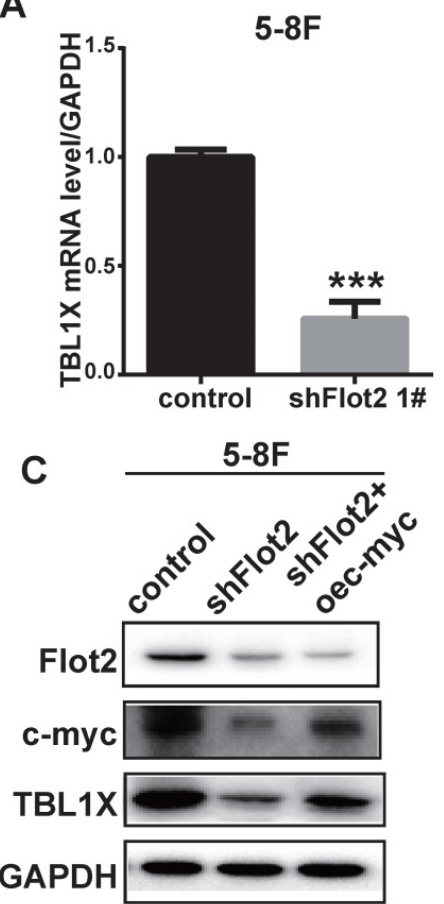

$\mathbf{F}$
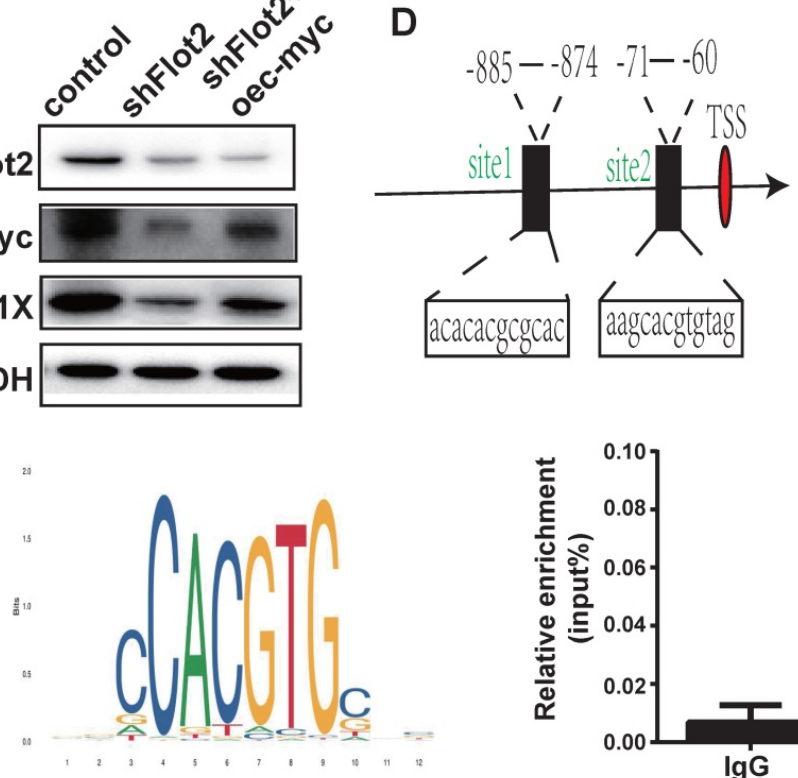

B
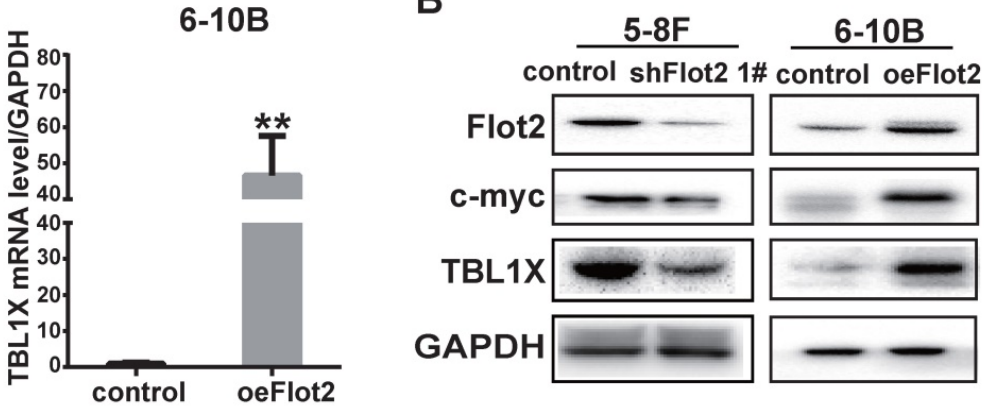

E

M Input lgG c-myc $M$
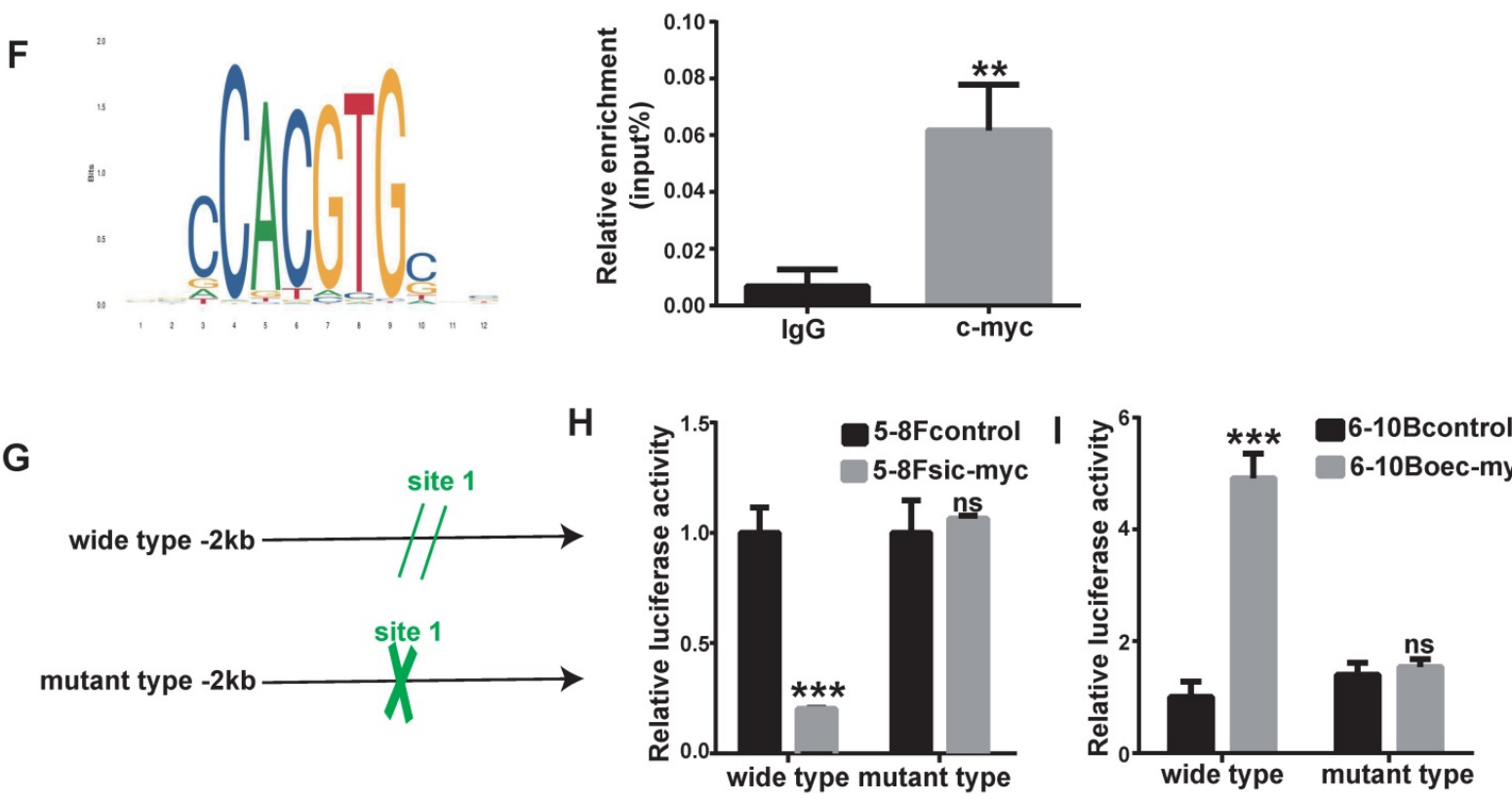

Figure 6. TBL1X transcription is regulated by Flot2 via c-myc. (A) qRT-PCR results showed that Flot2 regulated the mRNA level of TBL1X. (B) Western blotting results showed that Flot2 regulated the expression of c-myc and TBL1X. (C) After overexpression of c-myc in 5-8FshFlot2 cells, the TBL1X protein level increased compared to $5-8$ FshFlot2 cells. (D) The c-myc binding sites in TBL1X promoter region were predicted by using JASPAR. (E, F) ChIP assay identified the binding between c-myc and TBL1X promoter DNA fragment. $(G)$ The wild type and mutant $P$ GL3basic-TBLIX promoter-reporter constructs. (H, I) Relative luciferase activities in 5-8F cells treated with si-c-myc $(\mathrm{H})$ and in 6-10B cells treated with oe-c-myc (I) after transfection of pGL3basic-TBLIX plasmids containing wild type or mutant TBL1X promoter region. The results are shown as means \pm SD. $* P<0.05, * * P<0.01, * * * P<0.001$.

\section{Discussion}

Metastasis is the major cause of treatment failure of NPC patients; thus, investigating the potential molecular mechanisms underlying NPC metastasis and identifying novel effective targets are critical to improving treatment outcomes. In the current study, we identified a novel TBL1X-Flot2 feedback axis that contributed to NPC metastasis. High expression levels of TBL1X and Flot2 predicted unfavorable outcomes in NPC cases. Supported by the experimental data, we demonstrated the clinical significance of TBL1X and Flot2 as biomarkers of NPC metastasis, which supports their high targeted therapeutic value for NPC patients. 


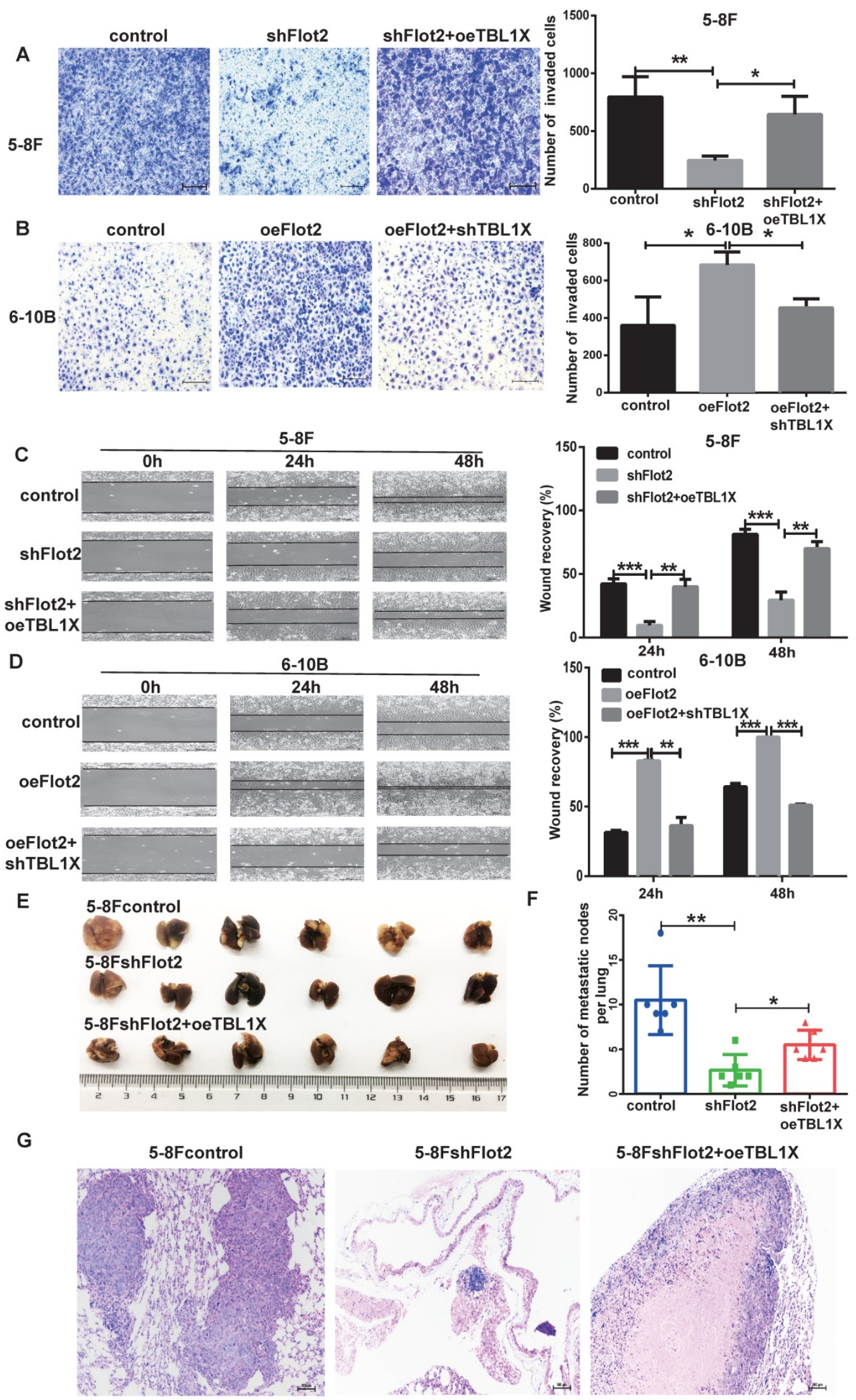

Figure 7. TBLIX rescues the effect of altered Flot2 expression on NPC cell migration and invasion. (A) Matrigel invasion analysis showed that oeTBLIX enhanced the invasion of 5-8FshFlot2 cells. (B) shTBL1X inhibited the invasion of 6-10B-Flot2 cells. (C) Wound-healing assay showed that oeTBL1X enhanced the migration 5-8FshFlot2 cells. (D) shTBLIX inhibited the migration of 6-10B-Flot2 cells. (E) Nude mouse metastasis assay showed the lung metastases via tail vein injection of 5-8Fcontrol, 5-8FshFlot2 or 5-8FshFlot2+oeTBLIX cells $(n=6$, respectively). ( $F$ ) The number of lung metastases in mice. (G) Representative HE staining images of lung metastases resulting from $5-8$ Fcontrol, 5-8FshFlot2 or 5-8FshFlot2+oeTBL1X cell inoculations. The results are shown as means \pm SD. $* P<0.05, * * P<0.01, * * * P<0.001$. 


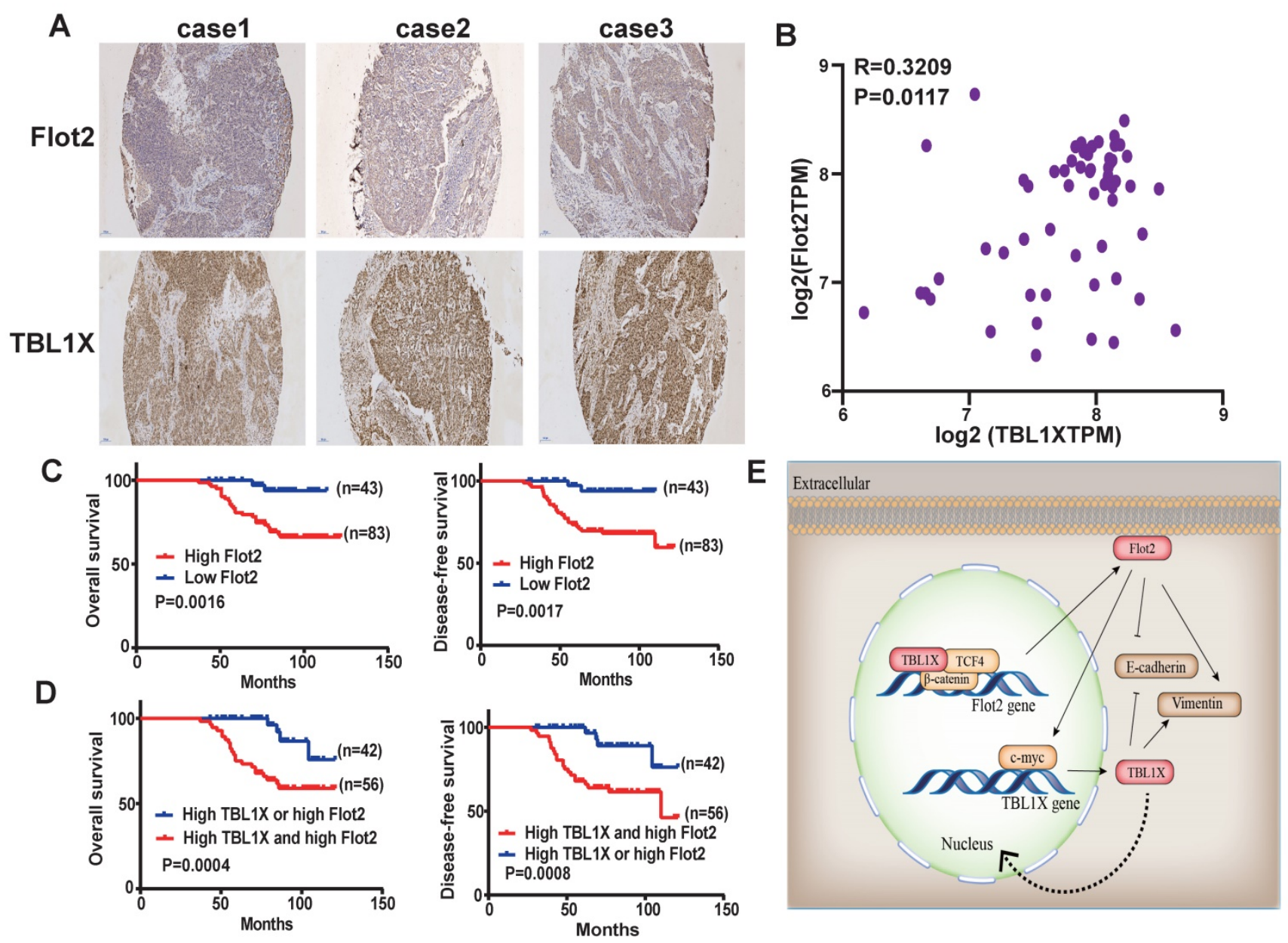

Figure 8. TBLIX expression is positively associated with Flot2 level in NPC. (A) Representative IHC images of TBL1X and Flot2 expression in NPC samples (Scale bar $=100 \mu \mathrm{m}$ ). (B) Positive correlation between TBLIX and Flot2 expression in NPC tissues of GEO batch. (C) Kaplan Meier survival analysis for NPC patients based on expression levels of Flot2. (D) Kaplan Meier survival analysis for NPC patients based on expression levels of both TBL1X and Flot2. (E) Model for tumor promotion role of TBL1X-Flot2 feedback axis and the underlying mechanisms in NPC.

Our results showed that TBL1X acted as an oncogene in NPC cases. TBL1X exhibited a higher expression level in metastatic NPC tissues. Upregulated TBL1X expression promoted NPC metastasis. As a transcriptional cofactor, TBL1X acts as a master regulator of Wnt signaling by forming complexes with $\beta$-catenin and the TCF family, and guides complexes to the promoter regions of target genes for oncogenesis [23]. Our results indicated that TBL1X regulated Flot2 expression by binding to Flot2 endogenous promoter with TCF4. Meanwhile, a previous study showed that TBL1X regulated the stability of critical oncogenic proteins such as PLK1, MYC, and beclin-1 through its interaction with an SKP1-CUL1-F-box (SCF) protein super-complex without $\beta$-catenin [24]. BC2059, also known as Tegavivint, a disruptor of TBL1X binding, can overcome resistance to BET inhibitors and improve survival in human osteosarcoma and AML [8, 25], which suggests that BC2059 may be an effective drug for relevant tumor treatment. TBL1X is also necessary for RELA recruitment to NF-kB-targeted gene promoters in response to TNF-a [26], which suggests an extra function of TBL1X as a transcriptional activator. In another study, SUMOylation of TBL1 enhances relation with NF-KB in response to TNF- $\alpha$ to promote the expression of targeted genes [27]. A similar study has shown that TBL1X suppresses E-cadherin expression by interacting with TWIST, thereby promoting tumor metastasis [7]. Therefore, identifying proteins interacting with TBL1X may help elucidate novel functions and mechanisms of TBL1X in tumors. In this study, we first found TBL1X regulated Flot2 expression at both mRNA and protein levels and subsequently discovered TCF4 was one of the potential transcription factors of Flot2. Our results demonstrated that TBL1X regulated Flot2 expression by interacting with TCF4.

Our cDNA microarray (GSE67456) and experimental data proved Flot2 regulated TBL1X expression; thus, the TBL1X-Flot2 axis forms a positive feedback axis and promotes NPC metastasis. 
Moreover, Flot2 regulated TBL1X expression through c-myc. Liu et al. found that Flot2 regulated c-myc expression by suppressing miR-33b-5p [28]. As a membrane protein, Flot2 promotes multiple tumor progression processes through different pathways such as Raf/MEK/ERK1/2[18], NF-kB, PI3K/ AKT [21], and TGF- $\beta[16,29]$ pathways. However, the exact mechanisms by which Flot2 activates these oncogenic functions and pathways require further research. Our previous study showed that Flot2 and PLCD3 interact with each other, this complex promotes NPC progression, and PLCD3 is a pivotal enzyme in the phosphoinositide pathway [30]. Flotillin-1 (Flot1) is a highly conserved homologous protein of Flot2, and these two proteins form heterotetramers on the cytoplasmic side of the plasma membrane [12]. Sumoylation of Flot1 facilitates its nuclear translocation, stabilizes Snail, and promotes the Snail-mediated tumor EMT process [31]. This suggests that post-translational modifications could also affect the functions of Flot2 in diseases. In addition to TBL1X, Flot2 is also regulated by several miRNAs, such as miR-34a, miR-133, and miR-138 et al[32-34]. In a word, the complex relation between TBL1X and Flot2 may provide the rationale for combination strategies for NPC therapy.

In summary, the TBL1X-Flot2 axis forms a positive feedback loop and promotes metastasis in NPC. High expression levels of both genes are associated with the poor prognosis of NPC cases. Therefore, the TBL1X-Flot2 feedback axis may be a potential target and valuable for improving NPC treatment.

\section{Abbreviations}

BLCA: bladder urothelial carcinoma; BRCA: breast invasive carcinoma; CHOL: cholangiocarcinoma; COAD: colon adenocarcinoma; ESCA: esophageal carcinoma; GBM: glioblastoma multiforme; HNSC: head and neck squamous cell carcinoma; KICH: kidney chromophobe; KIRC: kidney renal clear cell carcinoma; KIRP: kidney renal papillary cell carcinoma; LGG: brain lower grade glioma; LIHC: liver hepatocellular carcinoma; LUAD: lung adenocarcinoma; LUSC: lung squamous cell carcinoma; PAAD: pancreatic adenocarcinoma; PRAD: prostate adenocarcinoma; READ: rectum adenocarcinoma; STAD: stomach adenocarcinoma; THCA: thyroid carcinoma; UCEC: uterine corpus endometrial carcinoma; TBL1X: Transducin $\beta$-like protein $1 \mathrm{X}$; EMT: epithelial-mesenchymal transition; ZEB1: zinc finger E-box binding homeobox 1; CDH1: cadherin 1; TCF4: transcription factor 4; SSH: suppression subtractive hybridization; IHC: immunohistochemistry; HE: hematoxylin and eosin;
qRT-PCR: quantitative real-time reverse transcription PCR; ChIP: chromatin immunoprecipitation assay; Co-IP: Co-immunoprecipitation; OS: overall survival; DFS: disease-free survival; GSEA: gene set enrichment analysis.

\section{Supplementary Material}

Supplementary figures.

https://www.ijbs.com/v18p1134s1.pdf

\section{Acknowledgments}

This study was supported by the National Natural Science Foundation of China (81773179, 81272972, 81872202), Natural Science Foundation of Hunan Province (2020JJ4771), Hunan Provincial Science and Technology Project (2014FJ6006), Natural Science Foundation of Guangdong Province (2018A030313778), Independent Exploration and Innovation Project of Central South University (2021zzts0929).

\section{Author Contributions}

Caiping Ren, Xingjun Jiang and Jianxiong Li supervised entire project and involved in the designing of all experiments; Hongjuan $\mathrm{Xu}$, Xuejun Yan wrote the manuscript; Hongjuan $\mathrm{Xu}$, Xuejun Yan, Jin Zhao, Kefan Zhou, Xiwu Liu, Li Ye, Quanwei Zhou, and Shasha Li performed experiments; Yuanbo Kang drew the model picture; Hecheng Zhu, Lei Wang, Bin Zhu, and Ming Zhao performed partial data analyses; Caiping Ren, Weidong Liu revised the manuscript; Weiren Luo evaluated the IHC results.

\section{Competing Interests}

The authors have declared that no competing interest exists.

\section{References}

1. Sung H, Ferlay J, Siegel RL, Laversanne M, Soerjomataram I, Jemal A, et al. Global Cancer Statistics 2020: GLOBOCAN Estimates of Incidence and Mortality Worldwide for 36 Cancers in 185 Countries. CA Cancer J Clin. 2021; 71: 209-49

2. Yu-Pei Chen ATCC, Quynh-Thu Le, Pierre Blanchard, Ying Sun, Jun Ma. Nasopharyngeal carcinoma. Lancet. 2019; 394: 64-80.

3. Yang Q, Cao S-M, Guo L, Hua Y-J, Huang P-Y, Zhang X-L, et al. Induction chemotherapy followed by concurrent chemoradiotherapy versus concurrent chemoradiotherapy alone in locoregionally advanced nasopharyngeal carcinoma: long-term results of a phase III multicentre randomised controlled trial. Eur J cancer. 2019; 119: 87-96.

4. Kang Y, He W, Ren C, Qiao J, Guo Q, Hu J, et al. Advances in targeted therapy mainly based on signal pathways for nasopharyngeal carcinoma. Signal Transduct Target Ther. 2020; 5: 245 .

5. M T Bassi RSR, B Caciotti, I M Winship, A De Grandi, M Riboni, P L Townes, P Beighton, A Ballabio, G Borsani. X-linked late-onset sensorineural deafness caused by a deletion involving OA1 and a novel gene containing WD-40 repeats. Am J Hum Genet. 1999; 64: 13.

6. Yoon H-G, Chan DW, Huang Z-Q, Li J, Fondell JD, Qin J, et al. Purification and functional characterization of the human $\mathrm{N}-\mathrm{CoR}$ complex: the roles of HDAC3, TBL1 and TBLR1. EMBO J. 2003; 22: 1336-46.

7. Rivero S, Gomez-Marin E, Guerrero-Martinez JA, Garcia-Martinez J, Reyes JC. TBL1 is required for the mesenchymal phenotype of transformed breast cancer cells. Cell Death Dis. 2019; 10: 95. 
8. Nomura M, Rainusso N, Lee $\mathrm{Y}-\mathrm{C}$, Dawson B, Coarfa C, Han R, et al. Tegavivint and the $\beta$-Catenin/ALDH Axis in Chemotherapy-Resistant and Metastatic Osteosarcoma. J Natl Cancer Inst. 2019; 111: 1216-27.

9. Tan M, Gong H, Wang J, Tao L, Xu D, Bao E, et al. SENP2 regulates MMP13 expression in a bladder cancer cell line through SUMOylation of TBL1/TBLR1. Sci Rep. 2015; 5: 13996.

10. Stoy C, Sundaram A, Rios Garcia M, Wang X, Seibert O, Zota A, et al. Transcriptional co-factor Transducin beta-like (TBL) 1 acts as a checkpoint in pancreatic cancer malignancy. EMBO Mol Med. 2015; 7: 1048-62.

11. Zhao F, Zhang J, Liu Y-S, Li L, He Y-L. Research advances on flotillins. Virol J. 2011; 8: 479 .

12. Kwiatkowska K, Matveichuk OV, Fronk J, Ciesielska A. Flotillins: At the Intersection of Protein S- Palmitoylation and Lipid-Mediated Signaling. Int J Mol Sci. 2020; 21: 2283

13. Huang S, Zheng S, Huang S, Cheng H, Lin Y, Wen Y, et al. Flot2 targeted by miR-449 acts as a prognostic biomarker in glioma. Artif Cells Nanomed Biotechnol. 2019; 47: 250-5.

14. Song $\mathrm{T}, \mathrm{Hu} \mathrm{Z}$, Liu J, Huang $\mathrm{W}$. FLOT2 upregulation promotes growth and invasion by interacting and stabilizing EphA2 in gliomas. Biochem Biophys Res Commu. 2021; 548: 67-73.

15. Wang X, Yang $\mathrm{O}$, Guo L, Li X-H, Zhao X-H, Song L-B, et al. Flotillin-2 is associated with breast cancer progression and poor survival outcomes. J Transl Med. 2013; 11: 190.

16. Zhao L, Lin L, Pan C, Shi M, Liao Y, Bin J, et al. Flotillin-2 promotes nasopharyngeal carcinoma metastasis and is necessary for the epithelial-mesenchymal transition induced by transforming growth factor- $\beta$. Oncotarget. 2015; 6: 9781-93.

17. Ma DM, Sun D, Wang J, Jin DH, Li Y, Han YE. Long non-coding RNA MIR4435-2HG recruits miR-802 from FLOT2 to promote melanoma progression. Eur Rev Med Pharmacol Sci. 2020; 24: 2616- 24.

18. Wang C-H, Zhu X-D, Ma D-N, Sun H-C, Gao D-M, Zhang N, et al. Flot2 promotes tumor growth and metastasis through modulating cell cycle and inducing epithelial-mesenchymal transition of hepatocellular carcinoma. Am J Cancer Res. 2017; 7: 1068-83.

19. Wang Y-L, Yao W-J, Guo L, Xi H-F, Li S-Y, Wang Z-M. Expression of flotillin-2 in human non-small cell lung cancer and its correlation with tumor progression and patient survival. Int J Clin Exp Pathol. 2015; 8: 601- 7.

20. Wen Q, Alnemah MM, Luo J, Wang W, Chu S, Chen L, et al. FLOT-2 is an independent prognostic marker in oral squamous cell carcinoma. Int J Clin Exp Pathol. 2015; 8: 8236-43.

21. Liu J, Huang W, Ren C, Wen Q, Liu W, Yang X, et al. Flotillin-2 promotes metastasis of nasopharyngeal carcinoma by activating NF-kB and PI3K/Akt3 signaling pathways. Sci Rep. 2015; 5: 11614.

22. Zhou W, Feng X, Ren C, Jiang X, Liu W, Huang W, et al. Over-expression of BCAT1, a c-Myc target gene, induces cell proliferation, migration and invasion in nasopharyngeal carcinoma. Mol Cancer. 2013; 12: 53.

23. Li J, Wang CY. TBL1-TBLR1 and beta-catenin recruit each other to Wnt target-gene promoter for transcription activation and oncogenesis. Nat Cell Biol. 2008; 10: 160-U36.

24. Youssef Y, Karkhanis V, Chan WK, Jeney F, Canella A, Zhang X, et al. Transducin $\beta$-like protein 1 controls multiple oncogenic networks in diffuse large B-cell lymphoma. Haematologica. 2020; Online ahead of print.

25. Saenz DT, Fiskus W, Manshouri T, Mill CP, Qian Y, Raina K, et al. Targeting nuclear $\beta$-catenin as therapy for post-myeloproliferative neoplasm secondary AML. Leukemia. 2019; 33: 1373-86.

26. Ramadoss S, Li J, Ding X, Al Hezaimi K, Wang C-Y. Transducin $\beta$-like protein 1 recruits nuclear factor $\mathrm{KB}$ to the target gene promoter for transcriptional activation. Mol Cell Biol. 2011; 31: 924-34.

27. Park S-Y, Na Y, Lee M-H, Seo J-S, Lee Y-H, Choi K-C, et al. SUMOylation of TBL1 and TBLR1 promotes androgen-independent prostate cancer cell growth. Oncotarget. 2016; 7: 41110-22.

28. Liu $\mathrm{R}$, Liu J, Wu P, Yi H, Zhang B, Huang W. Flotillin-2 promotes cell proliferation via activating the c-Myc/BCAT1 axis by suppressing miR-33b-5p in nasopharyngeal carcinoma. Aging (Albany NY). 2021; 13: 8078-94.

29. Li O, Peng J, Li X, Leng A, Liu T. miR-449a targets Flot2 and inhibits gastric cancer invasion by inhibiting TGF- $\beta$-mediated EMT. Diagn Pathol. 2015; 10 : 202.

30. Liu W, Liu X, Wang L, Zhu B, Zhang C, Jia W, et al. PLCD3, a flotillin2-interacting protein, is involved in proliferation, migration and invasion of nasopharyngeal carcinoma cells. Oncol Rep. 2018; 39: 45-52.

31. Jang D, Kwon H, Choi M, Lee J, Pak Y. Sumoylation of Flotillin-1 promotes EMT in metastatic prostate cancer by suppressing Snail degradation. Oncogene. 2019; 38: 3248-60.

32. Liu R, Xie H, Luo C, Chen Z, Zhou X, Xia K, et al. Identification of FLOT2 as a novel target for microRNA-34a in melanoma. J Cancer Res Clin Oncol. 2015; 141: 993-1006.

33. Wei G, Xu Y, Peng T, Yan J. miR-133 involves in lung adenocarcinoma cell metastasis by targeting FLOT2. Artif Cells Nanomed Biotechnol. 2017; 46: 224-30.

34. Shi Y, Massagué J. Mechanisms of TGF-beta signaling from cell membrane to the nucleus. Cell. 2003; 113: 685-700. 